\title{
The Effects of a Remote Stereogenic Center in the Lewis Base Catalyzed Aldol Additions of Chiral Trichlorosilyl Enolates
}

\author{
Scott E. Denmark* and Shinji Fujimori \\ Roger Adams Laboratory, Department of Chemistry, University of Illinois, \\ Urbana-Champaign, Illinois 61801
}

\section{SUPPORTING INFORMATION}

\section{General Experimental}

${ }^{1} \mathrm{H},{ }^{13} \mathrm{C}$ and ${ }^{31} \mathrm{P}$ NMR spectra were recorded on Varian Unity-400 $\left(400 \mathrm{MHz}{ }^{1} \mathrm{H}, 100\right.$ $\left.\mathrm{MHz}{ }^{13} \mathrm{C}, 162 \mathrm{MHz}{ }^{31} \mathrm{P}\right)$, Unity-500 (500 MHz $\left.{ }^{1} \mathrm{H}, 125 \mathrm{MHz}{ }^{13} \mathrm{C}, 202 \mathrm{MHz}{ }^{31} \mathrm{P}\right)$ and Varian Unity Inova $500\left(500 \mathrm{MHz}{ }^{1} \mathrm{H}\right)$ spectrometers in deuterochloroform unless otherwise stated. Data are reported in the following order: chemical shift in $\mathrm{ppm}(\delta)$; multiplicities are indicated (br (broadened), s (singlet), d (doublet), t (triplet), q (quartet), m (multiplet)); coupling constants, $J$, are reported in hertz $(\mathrm{Hz})$; integration is provided; and assignment is indicated. Mass spectrometry was performed by the University of Illinois Mass Spectrometry Center. Electron impact (EI) spectra were performed on a Finnigan-MAT CH-5 spectrometer, chemical ionization (CI) spectra were obtained on a VG 70-VSE spectrometer using methane as the carrier gas, and field ionization (FI) spectra were obtained from VG 70-VSE A spectrometer. Data are reported in the form of $\mathrm{m} / \mathrm{z}$ (intensity relative to base peak $=100$ ). Infrared spectra (IR) were recorded on a Mattson Galaxy 5020 spectrophorometer. Peaks are reported in $\mathrm{cm}^{-1}$ with indicated intensities: s (strong, $67-100 \%$ absorption), m (medium, $34-66 \%$ ), w (weak, $0-33 \%$ ). Optical rotations were measured on a Jasco DIP-360 digital polarimeter and are reported in the standard format for $[\alpha]$. Elemental analyses were performed by the University of Illinois Microanalytical Service Laboratory.

Analytical thin-layer chromatography was performed on Merck silica gel plates with F254 indicator. Visualization was accomplished by UV light, iodine, potassium permanganate solution $\left(\mathrm{KMnO}_{4}\right)$, phosphomolibdic acid stain(PMA), p-anisaldehyde stain or dinitrophenylhydrazine (DNP) solution. Column chromatography was performed by the method 
of Still $^{1}$ with 32-63 mm silica gel (Merck). Bulb-to-bulb distillations were performed on a Buchi GKR-50 Kugelrohr and boiling points (bp) correspond to uncorrected, air bath temperatures. Analytical supercritical fluid chromatography (SFC) was performed on a Berger Instruments packed-column SFC with built in photometric detector. Analytical columns used were Daicel Chiralpak $\mathrm{AD}$ and $\mathrm{AS}$, Chiralcel OJ and OD and Regis $(R, R)$-Welk-01. Melting points (mp) were determined on a Thomas-Hoover melting point apparatus and are uncorrected.

All reactions were performed in oven $\left(140{ }^{\circ} \mathrm{C}\right)$ and/or flame-dried glassware under an atmosphere of dry nitrogen, unless otherwise stated. Solvents for extraction and chromatography were technical grade and distilled from the indicated drying agents: dichloromethane $\left(\mathrm{CH}_{2} \mathrm{Cl}_{2}\right)$, pentane, hexane: $\mathrm{CaCl}_{2}$; ethyl acetate (EtOAc): $\mathrm{K}_{2} \mathrm{CO}_{3}$; acetone and methanol $(\mathrm{MeOH})$ were reagent grade. $n$-Butyllithium was titrated according to the method of Gilman. "Brine" refers to a saturated solution of $\mathrm{NaCl}$.

\section{Literature Preparations}

The enolisation using LiTMP followed the procedure described by Collum et al. ${ }^{3}$ The enolization using DBU to synthesize $(Z)$-enol ethers followed the procedure described by Yamaguchi, et. al. ${ }^{4}$ tert-Butyltritylamine (TBTA) was prepared according to the procedure described by Corey, et. al. 5

\section{Experimental Procedures}

(S)-N-Methoxy- $N$-methyl-3-hydroxybutyramide $(13)^{6}$

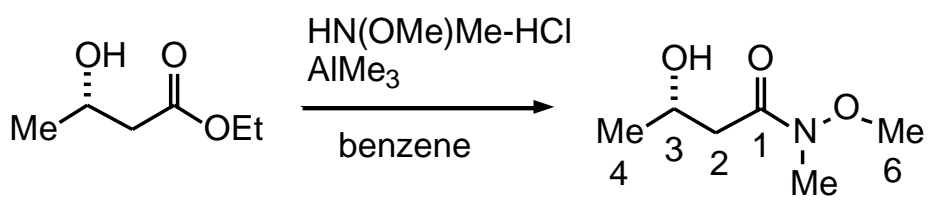

135

To a suspension of $\mathrm{N}, O$-dimethylhydroxylamine hydrochloride $(6.46 \mathrm{~g}, 66.2 \mathrm{mmol}, 2.5$ equiv) in $50 \mathrm{~mL}$ of benzene was added a solution of trimethylaluminum $(6.35 \mathrm{~mL}, 66.2 \mathrm{mmol}$, 2.5 equiv) in $40 \mathrm{~mL}$ benzene via cannula at $0{ }^{\circ} \mathrm{C}$. The reaction mixture was stirred at $\mathrm{rt}$ for $1 \mathrm{~h}$.

To the reaction mixture was added ethyl $(S)$-3-hydroxybutyrate $(3.50 \mathrm{~g}, 26.5 \mathrm{mmol})$, and the 
reaction mixture was heated to reflux for $3 \mathrm{~h}$. The reaction mixture was cooled to $0{ }^{\circ} \mathrm{C}$ and was quenched carefully with $50 \mathrm{~mL}$ of $1 \mathrm{M}$ aq. $\mathrm{HCl}$ solution. The layers were separated and the aqueous phase was extracted with $300 \mathrm{~mL}$ of $\mathrm{CH}_{2} \mathrm{Cl}_{2}$. The combined organic layers were dried $\left(\mathrm{MgSO}_{4}\right)$, filtered, and concentrated. Distillation of the residue afforded $3.32 \mathrm{~g}$ of $\mathbf{1 3}$ (22.6 mmol, $85 \%$ ) as a clear, colorless, viscous oil.

Data for 13:

bp: $\quad 82^{\circ} \mathrm{C}(0.5 \mathrm{mmHg})$

${ }^{1}$ HNMR: $\quad\left(500 \mathrm{MHz}, \mathrm{CDCl}_{3}\right)$

4.21 (m, $1 \mathrm{H}, \mathrm{HC}(3)) ; 3.87$ (d, J=1.7, $1 \mathrm{H}, \mathrm{OH}) ; 3.69$ (s, $\left.3 \mathrm{H}, \mathrm{H}_{3} \mathrm{C}(6)\right) ; 3.20$ (s, 3

$\left.\mathrm{H}, \mathrm{H}_{3} \mathrm{C}(5)\right) ; 2.69-2.42\left(\mathrm{ABX}, 2 \mathrm{H}, \mathrm{H}_{2} \mathrm{C}(2)\right) ; 1.24$ (d, $\left.J=6.3,3 \mathrm{H}, \mathrm{H}_{3} \mathrm{C}(4)\right)$

${ }^{13} \underline{\mathrm{C} \mathrm{NMR}}: \quad\left(126 \mathrm{MHz}, \mathrm{CDCl}_{3}\right)$

$173.77(\mathrm{C}(1)) ; 64.02(\mathrm{C}(3)) ; 61.15(\mathrm{C}(6)) ; 39.54(\mathrm{C}(5)) ; 31.71(\mathrm{C}(2)) ; 22.26$ $(\mathrm{C}(4))$

Opt. Rot.: $\quad[\alpha]_{\mathrm{D}}^{24}+57.4\left(\mathrm{c}=0.51, \mathrm{CHCl}_{3}\right)$

(S)- $N$-Methoxy- $N$-methyl-3-tert-butyldimethylsilyloxybutyramide (14)

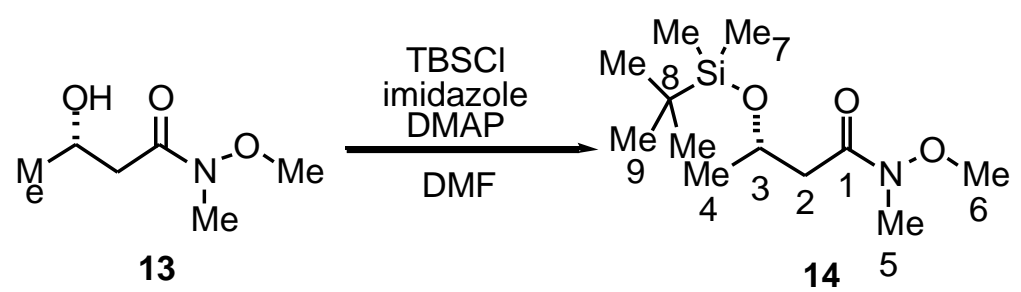

To a solution of hydroxy amide $13(3.24 \mathrm{~g}, 22.0 \mathrm{mmol})$ in $30 \mathrm{~mL}$ of DMF was added tertbutyldimethylchlorosilane (4.97 g, $33.0 \mathrm{mmol}, 1.5$ equiv), 4-dimethylaminopyridine (269 mg, $2.20 \mathrm{mmol}, 0.1$ equiv) and imidazole (4.49 $\mathrm{g}, 66.0 \mathrm{mmol}, 3$ equiv). The reaction mixture was stirred at $\mathrm{rt}$ for $36 \mathrm{~h}$ and then was diluted with $30 \mathrm{~mL}$ of water and was stirred for $1 \mathrm{~h}$. The product was extracted with $200 \mathrm{~mL}$ of pentane and the solution was dried $\left(\mathrm{MgSO}_{4}\right)$, filtered, and concentrated. The crude product was distilled using a Vigreux column to afford $5.75 \mathrm{~g}$ of silyloxy amide 14 (22.0 mmol, 99\%) as a clear, colorless oil. 
Data for 14:

bp: $\quad 88^{\circ} \mathrm{C}(0.1 \mathrm{mmHg})$

1ㅁN $\underline{\mathrm{HMR}}: \quad\left(500 \mathrm{MHz}, \mathrm{CDCl}_{3}\right)$

4.36 (sextd, $J=6.1,1.0,1 \mathrm{H}, \mathrm{HC}(3))$ ) 3.70 (s, $\left.3 \mathrm{H}, \mathrm{H}_{3} \mathrm{C}(6)\right)$ ) 3.17 (s, $3 \mathrm{H}$,

$\left.\mathrm{H}_{3} \mathrm{C}(5)\right) ; 2.96$ - 2.33 (ABX, $\left.2 \mathrm{H}, \mathrm{H}_{2} \mathrm{C}(2)\right) ; 1.22$ (d, $\left.J=6.1,3 \mathrm{H}, \mathrm{H}_{3} \mathrm{C}(4)\right) ; 0.87$ (s,

$\left.9 \mathrm{H}, \mathrm{H}_{3} \mathrm{C}(9)\right) ; 0.05\left(\mathrm{~d}, J=12.9,6 \mathrm{H}, \mathrm{H}_{3} \mathrm{C}(7)\right)$

${ }^{13} \underline{\mathrm{C} \mathrm{NMR}}: \quad\left(126 \mathrm{MHz}, \mathrm{CDCl}_{3}\right)$

$172.36(\mathrm{C}(1)) ; 65.98(\mathrm{C}(3)) ; 61.30(\mathrm{C}(6)) ; 41.67(\mathrm{C}(5)) ; 31.87(\mathrm{C}(2)) ; 25.77$

$(\mathrm{C}(9)) ; 24.14(\mathrm{C}(8)) ; 17.99(\mathrm{C}(4)) ;-4.74,-4.95$ (C(7))

IR: (neat)

2957 (s); 2931 (s); 2898 (m); 2857 (m); 1666 (s); 1464 (m); 1385 (m); 1255 (m); 1136 (m); 1123 (m); 1092 (s); 1068 (m); 1004(s)

MS: (FI)

$262.2\left(5, \mathrm{M}^{+}\right) ; 204.1(100)$

Opt. Rot.: $\quad[\alpha]_{\mathrm{D}}^{24}+24.1\left(\mathrm{c}=0.77, \mathrm{CHCl}_{3}\right)$

TLC: $\quad R_{f} 0.25$ (pentane/ether, 1/1) [silica gel, $\mathrm{KMnO}_{4}$ ]

Analysis: $\quad \mathrm{C}_{12} \mathrm{H}_{27} \mathrm{NO}_{3} \mathrm{Si}(261.43)$

Calcd: $\quad$ C, 55.13; $\quad$ H, 10.41; N, 5.36\%

Found: $\quad \mathrm{C}, 55.01 ; \quad \mathrm{H}, 10.61 ; \mathrm{N}, 5.46 \%$

\section{(S)- $N$-Methoxy- $N$-methyl-3-triisopropylsilyloxybutyramide (15)}

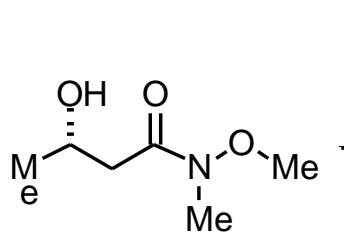

13

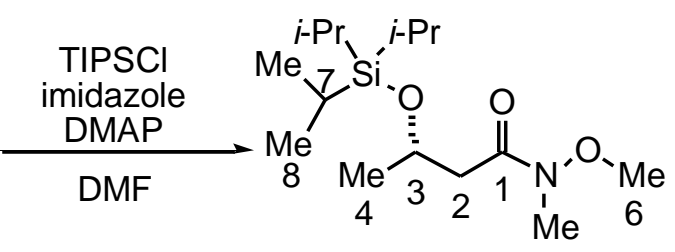

155

To a solution of hydroxy amide $13(3.60 \mathrm{~g}, 24.5 \mathrm{mmol})$ in $30 \mathrm{~mL}$ of DMF was added triisopropylchlorosilane (10.5 mL, $49.0 \mathrm{mmol}, 2$ equiv), 4-dimethylaminopyridine (399 $\mathrm{mg}, 2.45$ mmol, 0.1 equiv) and imidazole (5.00 g, $73.5 \mathrm{mmol}, 3$ equiv). The reaction mixture was stirred 
at $\mathrm{rt}$ for $48 \mathrm{~h}$ and then was diluted with $30 \mathrm{~mL}$ of water and was stirred for $1 \mathrm{~h}$. The product was extracted with $200 \mathrm{~mL}$ of pentane and the solution was dried $\left(\mathrm{MgSO}_{4}\right)$, filtered, and concentrated. The crude product was distilled using a Vigreux column to afford $6.90 \mathrm{~g}$ of silyloxy amide 15 (22.7 mmol, 93\%) as a clear, colorless oil.

Data for 15:

bp: $\quad 110^{\circ} \mathrm{C}(0.1 \mathrm{mmHg})$

${ }^{1} \underline{\mathrm{H} \text { NMR}}: \quad\left(500 \mathrm{MHz}, \mathrm{CDCl}_{3}\right)$

4.46 (sext, $J=6.1,1 \mathrm{H}, \mathrm{HC}(3)$ ); 3.69 (s, $\left.3 \mathrm{H}, \mathrm{H}_{3} \mathrm{C}(6)\right)$; 3.17 (s, $3 \mathrm{H}, \mathrm{H}_{3} \mathrm{C}(5)$ ); 2.78

- 2.44 (ABX, $\left.2 \mathrm{H}, \mathrm{H}_{2} \mathrm{C}(2)\right) ; 1.25$ (d, J = 5.8, $\left.3 \mathrm{H}, \mathrm{H}_{3} \mathrm{C}(4)\right)$; 1.08 - 1.04 (m, $21 \mathrm{H}$, $\left.\mathrm{H}_{3} \mathrm{C}(8), \mathrm{HC}(7)\right)$

${ }^{13} \mathrm{C} \mathrm{NMR}: \quad\left(126 \mathrm{MHz}, \mathrm{CDCl}_{3}\right)$

$164.78(\mathrm{C}(1)) ; 66.10(\mathrm{C}(3)) ; 61.50(\mathrm{C}(6)) ; 42.33(\mathrm{C}(5)) ; 24.52(\mathrm{C}(2)) ; 18.28$ $(\mathrm{C}(8)) ; 17.91(\mathrm{C}(4)) ; 12.57(\mathrm{C}(7))$

IR: (neat)

2962 (s); 2943 (s); 2895 (m); 2868 (s); 1668 (s); 1464 (m); 1414 (w); 1385 (m); 1178 (w); 1122 (m); 1093 (m); 1066 (m); 1009 (m)

MS: $\quad(\mathrm{FI})$

305.2 (13); $304.2\left(\mathrm{M}^{+}, 60\right) ; 261.2$ (19); 260.2 (100); 157.2 (13); 130.09 (19)

Opt. Rot.: $\quad[\alpha]_{\mathrm{D}}^{24}+14.5\left(\mathrm{c}=0.61, \mathrm{CHCl}_{3}\right)$

TLC: $\quad R_{f} 0.15$ (hexane/ether, 3/1) [silica gel, $\mathrm{KMnO}_{4}$ ]

Analysis: $\quad \mathrm{C}_{15} \mathrm{H}_{33} \mathrm{NO}_{3} \mathrm{Si}(303.51)$

Calcd: $\quad$ C, 59.36; $\quad$ H, 10.96; $\quad$ N, $4.61 \%$

Found: $\quad$ C, $59.17 ; \quad \mathrm{H}, 11.01 ; \quad \mathrm{N}, 4.67 \%$ 


\section{(S)-4-tert-Butyldimethylsilyloxy-2-pentanone (1)}

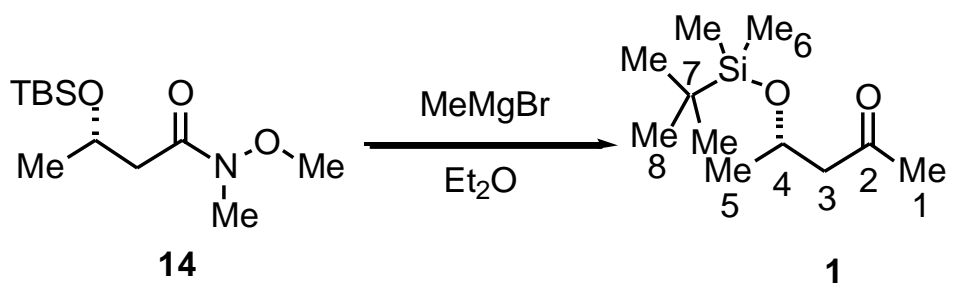

Amide $14(2.00 \mathrm{~g}, 7.65 \mathrm{mmol})$ was dissolved in $20 \mathrm{~mL}$ of $\mathrm{Et}_{2} \mathrm{O}$ at $0{ }^{\circ} \mathrm{C}$. To this solution was slowly added a solution of methylmagnesium bromide (3.0 $\mathrm{M}$ in $\mathrm{Et}_{2} \mathrm{O}, 3.83 \mathrm{~mL}, 11.5 \mathrm{mmol}$, 1.5 equiv). The reaction mixture was stirred at $0{ }^{\circ} \mathrm{C}$ for $1 \mathrm{~h}$, then was gradually allowed to warm to $\mathrm{rt}$ over $11 \mathrm{~h}$. The reaction mixture was quenched by slow addition of sat. aq. ammonium chloride solution (ca. $15 \mathrm{~mL}$ ). The layers were separated and the aqueous layer was extracted with $\mathrm{Et}_{2} \mathrm{O}(2 \times 50 \mathrm{~mL})$. The extracts were combined, dried $\left(\mathrm{MgSO}_{4}\right)$, filtered, and concentrated to give $842 \mathrm{mg}$ of an oil. The crude product was purified by chromatography (hexane/ether, $5 / 1$, $\left.\mathrm{SiO}_{2}\right)$ and afforded $1.39 \mathrm{~g}$ of $1(6.42 \mathrm{mmol}, 84 \%)$ as a clear, colorless liquid after Kugelrohr distillation.

Data for 1:

bp: $\quad 75^{\circ} \mathrm{C}(0.1 \mathrm{mmHg}, \mathrm{ABT})$

${ }^{1} \underline{\mathrm{H} \text { NMR}}: \quad\left(400 \mathrm{MHz}, \mathrm{CDCl}_{3}\right)$

4.28 (sextd, $J=6.0,1.0,1 \mathrm{H}, \mathrm{HC}(4)) ; 2.66-2.40\left(\mathrm{ABX}, 2 \mathrm{H}, \mathrm{H}_{2} \mathrm{C}(3)\right) ; 2.16$ (s, 3

$\left.\mathrm{H}, \mathrm{H}_{3} \mathrm{C}(1)\right) ; 1.17$ (d, $\left.J=6.1,3 \mathrm{H}, \mathrm{H}_{3} \mathrm{C}(5)\right)$; 0.86 (s, $9 \mathrm{H}, \mathrm{H}_{3} \mathrm{C}(8)$ ); 0.06, 0.03 (s, 6 $\left.\mathrm{H} \mathrm{H}_{3} \mathrm{C}(6)\right)$

${ }^{13}$ C NMR: $\quad\left(101 \mathrm{MHz}, \mathrm{CDCl}_{3}\right)$

208.15 (C(2)); 65.64 (C(4)); 53.13 (C(3)); 31.69 (C(1)); 25.77 (C(8)); 24.02 $(\mathrm{C}(7)) ; 17.96(\mathrm{C}(5)) ;-4.52,-5.01(\mathrm{C}(6))$

IR: (neat) 2957 (s); 2931 (s); 2896 (m); 2858 (s); 1720 (s); 1374 (m); 1360 (m); 1255 (s); 1135 (s); 1091 (s); 1022 (s)

$\underline{\mathrm{MS}}: \quad(\mathrm{FI})$

$217.7\left(4, \mathrm{M}^{+}\right) ; 184.2(2) ; 168.1(2) ; 159.0(100) ; 114.1$ (2) 
Opt. Rot.: $\quad[\alpha]_{\mathrm{D}}^{24}+32.5\left(\mathrm{c}=0.70, \mathrm{CHCl}_{3}\right)$

TLC: $\quad R_{f} 0.18$ (hexane/ether, 5/1) [silica gel, $\mathrm{KMnO}_{4}$ ]

Analysis: $\quad \mathrm{C}_{11} \mathrm{H}_{24} \mathrm{O}_{2} \mathrm{Si}(216.39)$
Calcd:
C, 61.05;
H, $11.18 \%$
Found:
C, 61.14;
$\mathrm{H}, 11.41 \%$

(S)-4-Triisopropylsilyloxy-2-pentanone (2)

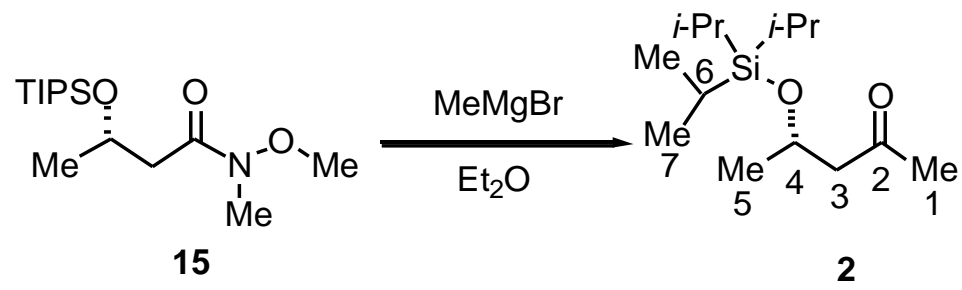

Amide 15 (1.23 g, $4.05 \mathrm{mmol})$ was dissolved in $10 \mathrm{~mL}$ of $\mathrm{Et}_{2} \mathrm{O}$ at $0{ }^{\circ} \mathrm{C}$. To this solution was slowly added a solution of methylmagnesium bromide (3.0 $\mathrm{M}$ in $\mathrm{Et}_{2} \mathrm{O}, 2.03 \mathrm{~mL}, 6.08 \mathrm{mmol}$, 1.5 equiv). The reaction mixture was stirred at $0{ }^{\circ} \mathrm{C}$ for $1 \mathrm{~h}$, then was gradually warmed over 11 $\mathrm{h}$ to $\mathrm{rt}$. The reaction mixture was quenched by slow addition of sat. aq. ammonium chloride solution (ca. $10 \mathrm{~mL}$ ). The layers were separated and the aqueous layer was extracted with $\mathrm{Et}_{2} \mathrm{O}$ $(2 \times 50 \mathrm{~mL})$. The extracts were combined, dried $\left(\mathrm{MgSO}_{4}\right)$, filtered, and concentrated. The crude product was purified by chromatography (hexane/ether, 5/1, $\mathrm{SiO}_{2}$ ) and afforded $811 \mathrm{mg}$ of 2 (3.14 mmol, 77\%) as a clear, colorless liquid.

Data for 2:

bp: $\quad 75^{\circ} \mathrm{C}(0.1 \mathrm{mmHg})$

1슬 $\quad\left(500 \mathrm{MHz}, \mathrm{CDCl}_{3}\right)$

4.40 (sext, $J=6.1,1 \mathrm{H}, \mathrm{HC}(4)) ; 2.70-2.51$ (ABX, $2 \mathrm{H}, \mathrm{H}_{2} \mathrm{C}(3)$ ); 2.17 (s, $3 \mathrm{H}$, $\left.\mathrm{H}_{3} \mathrm{C}(1)\right) ; 1.21$ (d, J=6.1, $\left.3 \mathrm{H}, \mathrm{H}_{3} \mathrm{C}(5)\right) ; 1.07-1.00\left(\mathrm{~m}, 21 \mathrm{H}, \mathrm{H}_{3} \mathrm{C}(7), \mathrm{HC}(6)\right)$

${ }^{13} \underline{\mathrm{C} \mathrm{NMR}}: \quad\left(126 \mathrm{MHz}, \mathrm{CDCl}_{3}\right)$

$208.0(\mathrm{C}(2)) ; 65.39$ (C(4)); 53.57 (C(3)); 31.49 (C(1)); 24.04 (C(5)); 18.06 (C(7)); $12.33(\mathrm{C}(6))$ 
IR: (neat)

2945 (s); 2894 (m); 2868 (s); 1720 (s); 1464 (m); 1372 (m); 1246 (w); 1133 (s); $1098(\mathrm{~m}) ; 1023(\mathrm{~m})$

MS: $\quad(\mathrm{FI})$

$258.1\left(2, \mathrm{M}^{+}\right) ; 217.1(5) ; 216.1(17) ; 215.1(100)$

Opt. Rot.: $\quad[\alpha]_{\mathrm{D}}^{24}+11.3\left(\mathrm{c}=0.63, \mathrm{CHCl}_{3}\right)$

TLC: $\quad R_{f} 0.35$ (hexane/ether, 5/1) [silica gel, $\mathrm{KMnO}_{4}$ ]

Analysis: $\quad \mathrm{C}_{14} \mathrm{H}_{20} \mathrm{O}_{2} \mathrm{Si}(258.47)$

Calcd: $\quad$ C, 65.06; H, $11.70 \%$

Found: $\quad$ C, $65.21 ; \quad H, 12.01 \%$

(4S)-4-(tert-Butyldimethylsilyloxy)-2-trimethylsilyloxy-1-pentene (16)

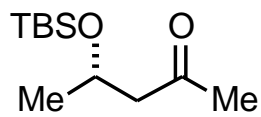

1

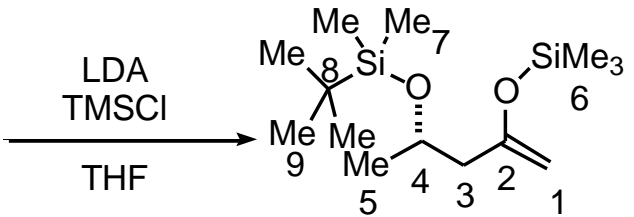

16

A solution of $n$-butyllithium (1.54 M in hexane, $4.20 \mathrm{~mL}, 6.47 \mathrm{mmol}, 1.4$ equiv) was added dropwise to a solution of diisopropylamine $(0.971 \mathrm{~mL}, 6.93 \mathrm{mmol}, 1.5$ equiv) in $15 \mathrm{~mL}$ of $\mathrm{THF}$ at $0{ }^{\circ} \mathrm{C}$. The reaction mixture was stirred at $0{ }^{\circ} \mathrm{C}$ for $10 \mathrm{~min}$ then was cooled to $-78{ }^{\circ} \mathrm{C}$. To the reaction mixture was added trimethylchlorosilane $(0.879 \mathrm{~mL}, 6.93 \mathrm{mmol}, 1.5 \mathrm{equiv})$ and ketone $1(1.00 \mathrm{~g}, 4.62 \mathrm{mmol})$ and the reaction mixture was stirred for $30 \mathrm{~min}$. The reaction mixture was gradually warmed to $0{ }^{\circ} \mathrm{C}$ and was quenched with $20 \mathrm{~mL}$ of cold, deionized water. The layers were separated and the aqueous layer was extracted with pentane $(2 \times 30 \mathrm{~mL})$. The combined extracts were washed with $20 \mathrm{~mL}$ of sat. aq. copper sulfate solution, $15 \mathrm{~mL}$ of water and $20 \mathrm{~mL}$ of brine. The combined organic extracts were dried $\left(\mathrm{Na}_{2} \mathrm{SO}_{4}\right)$, filtered, and were concentrated to give $1.61 \mathrm{~g}$ of an oil. Kugelrohr distillation afforded $1.18 \mathrm{~g}$ of enolate $\mathbf{1 6}$ (4.09 mmol, 89\%) as clear, colorless oil. 


\section{Data for 16:}

bp: $\quad 75^{\circ} \mathrm{C}(0.5 \mathrm{mmHg}, \mathrm{ABT})$

1ㅁN $\underline{\mathrm{HMR}}: \quad\left(500 \mathrm{MHz}, \mathrm{CDCl}_{3}\right)$

4.04 (d, $J=7.6,2 \mathrm{H}, \mathrm{H}_{2} \mathrm{C}(1)$ ); 4.04 (sext, $J=6.5,1 \mathrm{H}, \mathrm{HC}(4)$ ); 2.26-2.01 (ABX,

$\left.2 \mathrm{H}, \mathrm{H}_{2} \mathrm{C}(3)\right) ; 1.15$ (d, J = 6.1, $\left.3 \mathrm{H}, \mathrm{H}_{3} \mathrm{C}(5)\right)$; 0.88 (s, $\left.9 \mathrm{H}, \mathrm{H}_{3} \mathrm{C}(9)\right)$; 0.20 (s, $9 \mathrm{H}$,

$\left.\mathrm{H}_{3} \mathrm{C}(6)\right) ; 0.05$ (d, $\left.J=1.9,6 \mathrm{H}, \mathrm{H}_{3} \mathrm{C}(7)\right)$

${ }^{13}$ C NMR: $\quad\left(126 \mathrm{MHz}, \mathrm{CDCl}_{3}\right)$

$156.70(\mathrm{C}(2))$; 91.75 (C(1)); $66.41(\mathrm{C}(4)) ; 47.50$ (C(3)); 25.90 (C(9)); 23.36 $(\mathrm{C}(8)) ; 18.20(\mathrm{C}(5)) ; 0.05(\mathrm{C}(6)) ;-4.67,-4.83(\mathrm{C}(7))$

IR: (neat)

2959 (s); 2930 (m); 2898 (m); 2858 (m); 1660 (m); $1637(\mathrm{~m}) ; 1464(\mathrm{~m}) ; 1377$ (m); 1304 (m); 1254 (s); 1211 (m); 1128 (m); 1087 (m); 1014 (m)

MS: $\quad(\mathrm{FI})$

289.2 (61, M+); 273.1 (8); 254.2 (4); 231.1 (100); 199.1 (1); 185.1 (3); 159.1 (41);

$131.1(3) ; 115.1(6) ; 73.1(40)$

Opt. Rot.: $\quad[\alpha]_{\mathrm{D}}^{24}+6.81\left(\mathrm{c}=3.5, \mathrm{CHCl}_{3}\right)$

Analysis: $\quad$ Calc for $\mathrm{C}_{14} \mathrm{H}_{32} \mathrm{O}_{2} \mathrm{Si}_{2}$ (288.57)
Calcd:
C, 58.27;
$\mathrm{H}, 11.18 \%$

Found:

C, 58.15;

$\mathrm{H}, 11.41 \%$

\section{(4S)-4-Triisopropylsilyloxy-2-trimethylsilyloxy-1-pentene (17)}

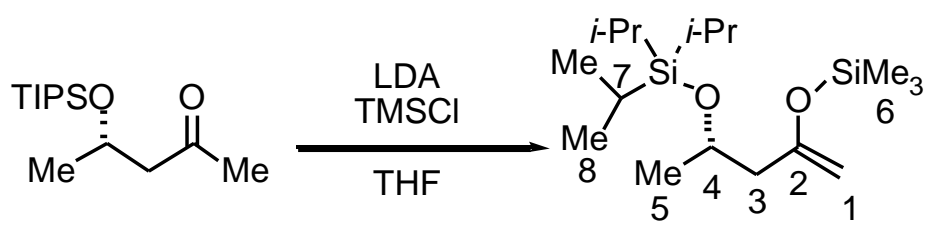

2

17

A solution of $n$-butyllithium (1.54 M in hexane, $2.29 \mathrm{~mL}, 3.53 \mathrm{mmol}, 1.4$ equiv) was added dropwise to a solution of diisopropylamine $(0.53 \mathrm{~mL}, 3.78 \mathrm{mmol}, 1.5$ equiv) in $10 \mathrm{~mL}$ of $\mathrm{THF}$ at $0{ }^{\circ} \mathrm{C}$. The reaction mixture was stirred at $0{ }^{\circ} \mathrm{C}$ for $10 \mathrm{~min}$ and then was cooled to $-78{ }^{\circ} \mathrm{C}$. 
To the reaction mixture was added trimethylchlorosilane $(048 \mathrm{~mL}, 3.78 \mathrm{mmol}, 1.5$ equiv) and ketone $2(651 \mathrm{mg}, 2.52 \mathrm{mmol})$ and the reaction mixture was stirred for $30 \mathrm{~min}$. The reaction mixture was gradually warmed to $0{ }^{\circ} \mathrm{C}$ and was quenched with $20 \mathrm{~mL}$ of cold, deionized water. The layers were separated and the aqueous layer was extracted with pentane $(2 \times 50 \mathrm{~mL})$. The combined extracts were washed with $20 \mathrm{~mL}$ of sat. aq. copper sulfate solution, $15 \mathrm{~mL}$ of water and $20 \mathrm{~mL}$ of brine. The organic extracts were dried $\left(\mathrm{Na}_{2} \mathrm{SO}_{4}\right)$, filtered, and concentrated to give $1.61 \mathrm{~g}$ of an oil. Kugelrohr distillation afforded $688 \mathrm{mg}$ of the enol ether 17 (2.08 mmol, $83 \%$ ) was obtained as clear, colorless oil.

\section{Data for 17:}

bp: $\quad 125^{\circ} \mathrm{C}(1.0 \mathrm{mmHg}, \mathrm{ABT})$

${ }^{1} \underline{\mathrm{H} \mathrm{NMR}}: \quad\left(500 \mathrm{MHz}, \mathrm{CDCl}_{3}\right)$

4.12 (sextd, $J=6.5,1.5,1 \mathrm{H}, \mathrm{HC}(4)) ; 4.04$ (d, $\left.J=11.7,2 \mathrm{H}, \mathrm{H}_{2} \mathrm{C}(1)\right) ; 2.38-2.02$

$\left(\mathrm{ABX}, 2 \mathrm{H}, \mathrm{H}_{2} \mathrm{C}(3)\right) ; 1.18\left(\mathrm{~d}, J=6.1,3 \mathrm{H}, \mathrm{H}_{3} \mathrm{C}(5)\right) ; 1.06-1.01(\mathrm{~m}, 21 \mathrm{H}$,

$\left.\mathrm{H}_{3} \mathrm{C}(8), \mathrm{HC}(7)\right) ; 0.19\left(\mathrm{~d}, J=1.9,6 \mathrm{H}, \mathrm{H}_{3} \mathrm{C}(6)\right)$

${ }^{13}$ C NMR: $\quad\left(126 \mathrm{MHz}, \mathrm{CDCl}_{3}\right)$

156.89 (C(2)); $91.63(\mathrm{C}(1)) ; 66.45$ (C(4)); 47.78 (C(3)); $23.17(\mathrm{C}(5)) ; 18.08$ $(\mathrm{C}(8)) ; 12.34(\mathrm{C}(7)) ;-0.01(\mathrm{C}(6))$

IR: (neat)

2962 (s); 2945 (s); 2885 (m); 2868 (s); 1659 (w); 1624 (w); 1464 (w); 1378 (w); 1303 (m); 1254 (m); 1211 (w); 1126 (m); 1090 (m); 1014 (s)

$\underline{\mathrm{MS}}: \quad$ (FI)

332.2 (21); $331.2\left(65, \mathrm{M}^{+}\right) ; 289.2$ (11); 288.2 (25); 287.2 (100); 201.2 (60); 73.0 (25)

Opt. Rot.: $\quad[\alpha]_{\mathrm{D}}^{24}+11.5\left(\mathrm{c}=0.95, \mathrm{CHCl}_{3}\right)$

Analysis: $\quad \mathrm{C}_{17} \mathrm{H}_{38} \mathrm{O}_{2} \mathrm{Si}_{2}(288.57)$

Calcd: $\quad$ C, $61.75 ; \quad H, 11.58 \%$

Found: $\quad$ C, $61.79 ; \quad H, 11.80 \%$ 
(4S)- 4-(tert-Butyldimethylsilyloxy)-2-trichlorosilyloxy-1-pentene (5)

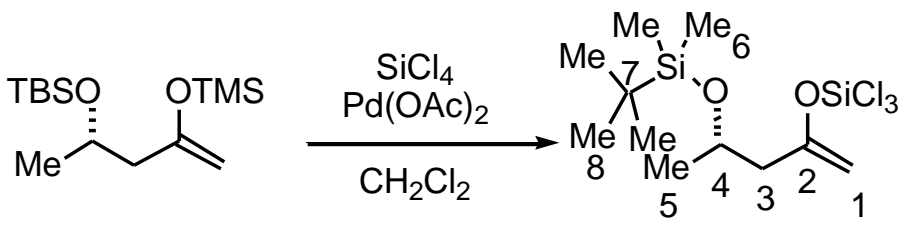

16

Silicon tetrachloride $(0.46 \mathrm{~mL}, 4.02 \mathrm{mmol}, 2.0$ equiv) was added to a solution of palladium(II) acetate $\left(22.6 \mathrm{mg}, 0.10 \mathrm{mmol}, 0.05\right.$ equiv) in $2.00 \mathrm{~mL}$ of $\mathrm{CH}_{2} \mathrm{Cl}_{2}$. To the reaction mixture was added trimethylsilyl enol ether $16(580 \mathrm{mg}, 2.01 \mathrm{mmol})$ using a cannula, and the reaction mixture was stirred at $\mathrm{rt}$ for $30 \mathrm{~min}$. Kugelrohr distillation of the reaction mixture afforded $526 \mathrm{mg}$ of trichlorosilyl enol ether 5 (1.50 mmol, 75\%) as clear, colorless liquid.

Data for 5:

bp: $\quad 125^{\circ} \mathrm{C}(1 \mathrm{mmHg}, \mathrm{ABT})$

1ㅁN $\underline{\mathrm{HMR}}: \quad\left(400 \mathrm{MHz}, \mathrm{CDCl}_{3}\right)$

$4.59\left(\mathrm{~d}, J=1.9,1 \mathrm{H}, \mathrm{H}_{2} \mathrm{C}(1)\right) ; 4.46$ (d, $\left.J=2.0,1 \mathrm{H}, \mathrm{H}_{2} \mathrm{C}(1)\right) ; 4.04$ (sext, $J=6.2,1$

$\mathrm{H}, \mathrm{HC}(4)) ; 2.35-2.16\left(\mathrm{ABX}, 2 \mathrm{H}, \mathrm{H}_{2} \mathrm{C}(3)\right) ; 1.18$ (d, J = 5.8, $\left.3 \mathrm{H}, \mathrm{H}_{3} \mathrm{C}(5)\right)$; 0.88 (s, $9 \mathrm{H}, \mathrm{H}_{3} \mathrm{C}(8) ; 0.06\left(\mathrm{~d}, J=3.6,6 \mathrm{H}, \mathrm{H}_{3} \mathrm{C}(6)\right)$

General Procedure I: (1R,5S)-5-tert-Butyldimethylsilyloxy-1-hydroxy-1-phenyl-3-hexanone (anti-9a)

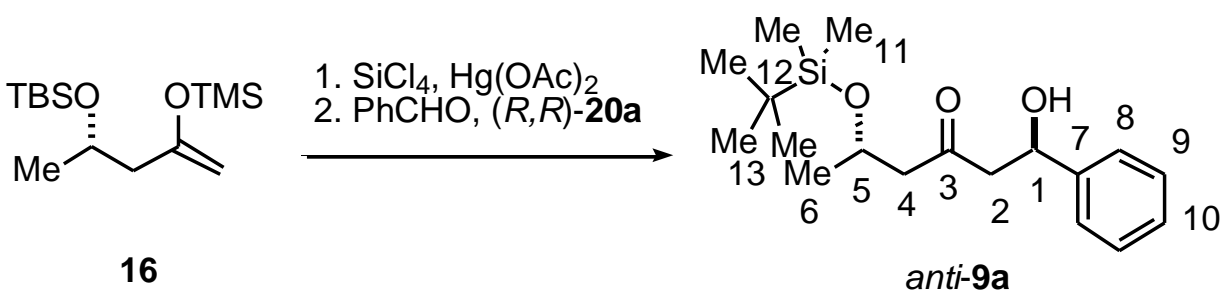

To a suspension of mercury(II) acetate $(3.2 \mathrm{mg}, 0.01 \mathrm{mmol}, 0.01$ equiv) in $1 \mathrm{~mL}$ of $\mathrm{CH}_{2} \mathrm{Cl}_{2}$ was added silicon tetrachloride $(0.23 \mathrm{~mL}, 2.0 \mathrm{mmol}, 2.0$ equiv) and trimethylsilyl enol ether 16 (289 mg, $1.00 \mathrm{mmol})$. The reaction mixture was stirred at $\mathrm{rt}$ for $30 \mathrm{~min}$. The excess 
silicon tetrachloride and the solvent were removed under vacuum. To the residue was added solution of the chiral phosphoramide $(R, R)-\mathbf{2 0 a}(36.9 \mathrm{mg}, 0.10 \mathrm{mmol}, 0.10$ equiv) in $2 \mathrm{~mL}$ of $\mathrm{CH}_{2} \mathrm{Cl}_{2}$ via cannula. The reaction mixture was cooled to $-78{ }^{\circ} \mathrm{C}$ before the addition of benzaldehyde $(102 \mu \mathrm{L}, 1.00 \mathrm{mmol})$. The reaction mixture was stirred at $-78{ }^{\circ} \mathrm{C}$ for $4 \mathrm{~h}$, and then was quenched by pouring it into a vigorously stirring solution of cold sat. aq. sodium bicarbonate solution $(5 \mathrm{~mL})$. The resulting slurry was stirred for $3 \mathrm{~h}$ and then was filtered through Celite. The layers were separated and the aqueous layer was extracted with $20 \mathrm{~mL}$ of $\mathrm{CH}_{2} \mathrm{Cl}_{2}$. The combined organic extracts were washed with $5 \mathrm{~mL}$ of brine, dried $\left(\mathrm{Na}_{2} \mathrm{SO}_{4}\right)$, filtered, and concentrated. The crude product was purified by chromatography (pentane/ether, $3 / 1, \mathrm{SiO}_{2}$ ) to $198 \mathrm{mg}$ of give 9a $(0.61 \mathrm{mmol}, 61 \%)$ as viscous, colorless oil.

\section{Data for anti-9a:}

${ }^{1} \underline{\mathrm{H} \mathrm{NMR}}: \quad\left(500 \mathrm{MHz}, \mathrm{CDCl}_{3}\right)$

7.35 - 7.34 (m, 4 H, HC(8), HC(9)); 7.29 - 7.26 (m, $1 \mathrm{H}, \mathrm{HC}(10)) ; 5.15$ (dt, J = 8.6, 3.5, 1H, HC(1)); 4.32 (sext, $J=6.1,1 \mathrm{H}, \mathrm{HC}(5)) ; 3.36$ (d, $J=3.2,1 \mathrm{H}, \mathrm{OH})$; $2.92-2.82\left(\mathrm{ABX}, 2 \mathrm{H}, \mathrm{H}_{2} \mathrm{C}(2)\right) ; 2.67-2.41\left(\mathrm{ABX}, 2 \mathrm{H}, \mathrm{H}_{2} \mathrm{C}(4)\right) ; 1.17$ (d, $J=$ $\left.6.2,3 \mathrm{H}, \mathrm{H}_{3} \mathrm{C}(6)\right) ; 0.86$ (s, 9H, $\left.\mathrm{H}_{3} \mathrm{C}(13)\right) ; 0.05$ (d, $J=12.4,6 \mathrm{H}, \mathrm{H}_{3} \mathrm{C}(11)$ )

${ }^{13}$ C NMR: $\quad\left(125 \mathrm{MHz}, \mathrm{CDCl}_{3}\right)$

$210.56(\mathrm{C}(3)) ; 142.71(\mathrm{C}(7)) ; 128.49$ (C(9)); 127.59 (C(10)); 125.59 (C(8)); 69.63 $(\mathrm{C}(5)) ; 65.64(\mathrm{C}(1)) ; 53.30(\mathrm{C}(4)) ; 52.85(\mathrm{C}(2)) ; 25.73(\mathrm{C}(13)) ; 23.99(\mathrm{C}(6))$; $17.89(\mathrm{C}(12)) ;-4.53,-5.00(\mathrm{C}(11))$

IR: (neat)

3438 (m, br); 3064 (w); 3032 (w); 2956 (s); 2929 (s); 2895 (m); 2850 (m); 1711 (s); 1495 (w); 1462 (m); 1377 (m); 1255 (m); 1134 (m); 1090 (m); 1066 (s); 1041 (m); 1007 (m)

MS: $\quad(\mathrm{FI})$

$323.1\left(5, \mathrm{M}^{+}\right) ; 265.1(100) ; 159.1(3) ; 106.1$ (3)

Opt. Rot.: $\quad[\alpha]_{\mathrm{D}}^{24}+47.1(\mathrm{c}=0.64$, EtOH $)$

TLC: $\quad R_{f} 0.10$ (pentane/ether, 3/1) [silicagel, $p$-anisaldehyde]

SFC: $\quad t_{\mathrm{R}} 4.695 \mathrm{~min}(\mathrm{OD}, 150 \mathrm{bar}, 3 \mathrm{~mL} / \mathrm{min}, 2 \% \mathrm{MeOH})$ 
Analysis: $\quad \mathrm{C}_{18} \mathrm{H}_{30} \mathrm{O}_{3} \mathrm{Si}(322.51)$

$\begin{array}{lll}\text { Calcd: } & \text { C, 67.03; } & \text { H, 9.38\% } \\ \text { Found: } & \text { C, 66.94; } & \text { H, } 9.52 \%\end{array}$

(1S,5S)-5-tert-Butyldimethylsilyloxy-1-hydroxy-1-phenyl-3-hexanone (syn-9a)

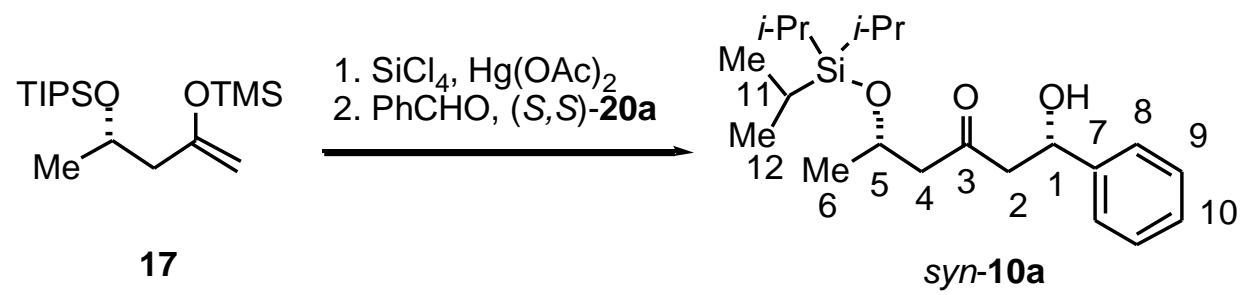

Following General Procedure I from $16(289 \mathrm{mg}, 1.00 \mathrm{mmol}), \mathrm{SiCl}_{4}(0.23 \mathrm{~mL}, 2.00$ $\mathrm{mmol}), \mathrm{Hg}(\mathrm{OAc})_{2}(3.2 \mathrm{mg}, 0.01 \mathrm{mmol})$, benzaldehyde $(102 \mu \mathrm{L}, 1.00 \mathrm{mmol}),(S, S)-20 \mathrm{a}(37 \mathrm{mg}$, $0.10 \mathrm{mmol})$ in $2 \mathrm{~mL}$ of $\mathrm{CH}_{2} \mathrm{Cl}_{2}$, was obtained $188 \mathrm{mg}(0.58 \mathrm{mmol}, 58 \%)$ of syn-9a as a clear, colorless, viscous oil after column chromatography (3/1 pentane/ether, $\mathrm{SiO}_{2}$ ).

Data for syn-9a:

${ }^{1}$ H NMR: $\quad\left(500 \mathrm{MHz}, \mathrm{CDCl}_{3}\right)$

7.36 - 7.34 (m, 4 H, $\mathrm{HC}(8), \mathrm{HC}(9)) ; 7.29-7.26$ (m, $1 \mathrm{H}, \mathrm{HC}(10)) ; 5.16-5.13$ (m, 1H, HC(1)); 4.32 (sext, $J=6.1,1 \mathrm{H}, \mathrm{HC}(5)$ ); 3.38 (d, $J=3.2,1 \mathrm{H}, \mathrm{OH}) ; 2.88$ -2.85 (ABX, 2H, $\mathrm{H}_{2} \mathrm{C}(2)$ ); $2.68-2.43\left(\mathrm{ABX}, 2 \mathrm{H}, \mathrm{H}_{2} \mathrm{C}(4)\right.$ ); 1.17 (d, $J=6.1,3 \mathrm{H}$, $\left.\mathrm{H}_{3} \mathrm{C}(6)\right) ; 0.86$ (s, 9H, $\left.\mathrm{H}_{3} \mathrm{C}(13)\right) ; 0.06\left(\mathrm{~d}, J=10.3,6 \mathrm{H}, \mathrm{H}_{3} \mathrm{C}(11)\right.$ )

${ }^{13}$ C NMR: $\quad\left(126 \mathrm{MHz}, \mathrm{CDCl}_{3}\right)$

$210.50(\mathrm{C}(3)) ; 142.74(\mathrm{C}(7)) ; 128.49(\mathrm{C}(9)) ; 127.59(\mathrm{C}(10)) ; 125.62(\mathrm{C}(8)) ; 69.80$ (C(5)); $65.47(\mathrm{C}(1)) ; 52.97(\mathrm{C}(4)) ; 52.79(\mathrm{C}(2)) ; 25.75$ (C(13)); 23.95 (C(6)); $17.91(\mathrm{C}(12)) ;-4.55,-4.97(\mathrm{C}(11))$

IR: (neat)

3441 (m, br); 3064 (w); 3032 (w); 2956 (s); 2930 (s); 2895 (m); 2858 (m); 1710 (m); 1495 (w); 1462 (m); 1376 (m); 1253 (m); 1132 (m); 1090 (m); 1068 (m); $1032(\mathrm{~m}) ; 1007$ (m) 
MS: (FI)

$323.2\left(5, \mathrm{M}^{+}\right) ; 265.1(100) ; 159.1(3) ; 106.1$ (3)

Opt. Rot.: $\quad[\alpha]_{\mathrm{D}}^{24}+1.61(\mathrm{c}=0.95$, EtOH $)$

TLC: $\quad R_{f} 0.11$ (pentane/ether, 3/1) [silica gel, $p$-anisaldehyde]

SFC: $\quad t_{\mathrm{R}} 4.984 \mathrm{~min}$ (OD, $150 \mathrm{bar}, 3 \mathrm{~mL} / \mathrm{min}, 2 \% \mathrm{MeOH}$ )

Analysis: $\quad \mathrm{C}_{18} \mathrm{H}_{30} \mathrm{O}_{3} \mathrm{Si}(322.51)$

Calcd: $\quad$ C, 67.03; H, $9.38 \%$

Found: $\quad$ C, $67.00 ; \quad H, 9.57 \%$

(1R,5S)-5-Triisopropylsilyloxy-1-hydroxy-1-phenyl-3-hexanone (anti-10a)

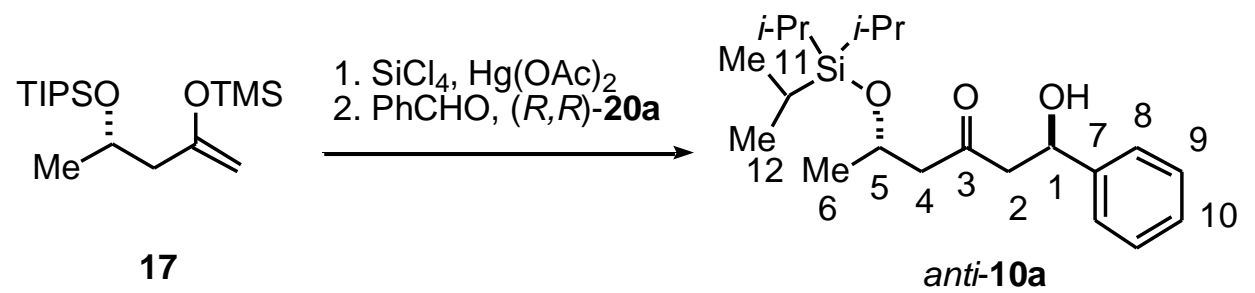

Following General Procedure I from $17(331 \mathrm{mg}, 1.00 \mathrm{mmol}), \mathrm{SiCl}_{4}(0.23 \mathrm{~mL}, 2.00$ $\mathrm{mmol}), \mathrm{Hg}(\mathrm{OAc})_{2}(3.2 \mathrm{mg}, 0.01 \mathrm{mmol})$, benzaldehyde $(102 \mu \mathrm{L}, 1.00 \mathrm{mmol}),(R, R)-\mathbf{2 0 a}(37 \mathrm{mg}$, $0.10 \mathrm{mmol})$ in $2 \mathrm{~mL}$ of $\mathrm{CH}_{2} \mathrm{Cl}_{2}$, was obtained $325 \mathrm{mg}(0.89 \mathrm{mmol}, 89 \%)$ of anti-10a as a clear, colorless, viscous oil after column chromatography (3/1 pentane/ether, $\left.\mathrm{SiO}_{2}\right)$.

Data for anti-10a:

${ }^{1} \underline{\mathrm{H} \mathrm{NMR}}: \quad\left(500 \mathrm{MHz}, \mathrm{CDCl}_{3}\right)$

7.37 (m, 4 H, HC(8), HC(9)); 7.30 (m, $1 \mathrm{H}, \mathrm{HC}(10))$; 5.17 (dt, J = 9.0, 3.1, 1H, $\mathrm{HC}(1)) ; 4.47$ (sext, $J=6.1,1 \mathrm{H}, \mathrm{HC}(5)) ; 3.35$ (d, $J=3.2,1 \mathrm{H}, \mathrm{OH}) ; 2.96-2.85$ $\left(\mathrm{ABX}, 2 \mathrm{H}, \mathrm{H}_{2} \mathrm{C}(2)\right) ; 2.73-2.52\left(\mathrm{ABX}, 2 \mathrm{H}, \mathrm{H}_{2} \mathrm{C}(4)\right) ; 1.24(\mathrm{~d}, J=6.1,3 \mathrm{H}$, $\left.\mathrm{H}_{3} \mathrm{C}(6)\right) ; 1.07$ (s, 21H, $\left.\mathrm{H}_{3} \mathrm{C}(12), \mathrm{HC}(11)\right)$ 
${ }^{13} \underline{\mathrm{C} N M R}: \quad\left(126 \mathrm{MHz}, \mathrm{CDCl}_{3}\right)$

$210.31(\mathrm{C}(3)) ; 142.72(\mathrm{C}(7)) ; 128.47(\mathrm{C}(9)) ; 127.58(\mathrm{C}(10)) ; 125.60(\mathrm{C}(8)) ; 69.69$ $(\mathrm{C}(1)) ; 65.40(\mathrm{C}(5)) ; 53.32(\mathrm{C}(4)) ; 52.76(\mathrm{C}(2)) ; 24.05(\mathrm{C}(6)) ; 18.05(\mathrm{C}(12))$; $12.35(\mathrm{C}(11))$

IR: (neat) 3441 (m, br); 2961 (s); 2944 (s); 2893 (s); 2867 (s); 1710 (s); 1463 (m); 1375 (m); 1130 (s); 1092 (s); 1066 (s); 1040 (m); 1013 (s)

MS: $\quad(\mathrm{FI})$

$365.2\left(2, \mathrm{M}^{+}\right) ; 321.0(100) ; 215.0$ (7); 143.7 (2); 106.0 (3)

Opt. Rot.: $\quad[\alpha]_{\mathrm{D}}^{24}+11.5(\mathrm{c}=0.43$, EtOH $)$

TLC: $\quad R_{f} 0.15$ (pentane/ether, 3/1) [silica gel, $p$-anisaldehyde]

SFC: $\quad t_{\mathrm{R}} 3.745 \mathrm{~min}(\mathrm{OD}, 150 \mathrm{bar}, 3 \mathrm{~mL} / \mathrm{min}, 5 \% \mathrm{MeOH})$

Analysis: $\quad \mathrm{C}_{21} \mathrm{H}_{36} \mathrm{O}_{3} \mathrm{Si}(364.59)$

Calcd: $\quad$ C, 69.18; H, $9.95 \%$

Found: $\quad \mathrm{C}, 69.04 ; \quad \mathrm{H}, 10.05 \%$

\section{(1S,5S)-5-Triisopropylsilyloxy-1-hydroxy-1-phenyl-3-hexanone (syn-10a)}

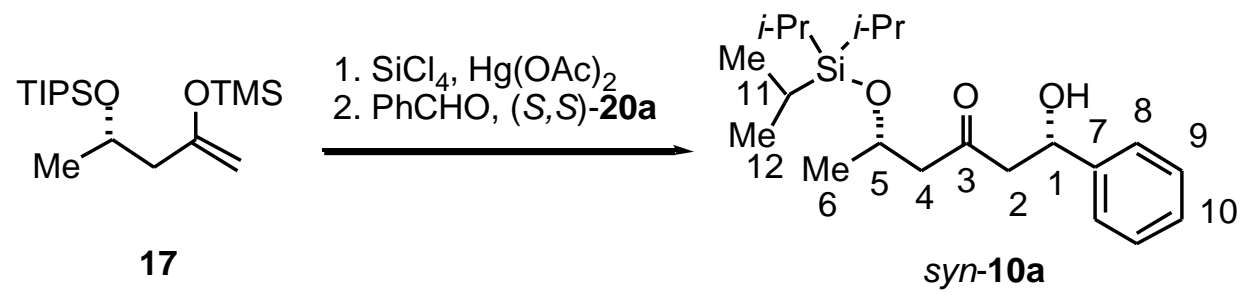

Following General Procedure I from 17 (331 mg, $1.00 \mathrm{mmol}), \mathrm{SiCl}_{4}(0.23 \mathrm{~mL}, 2.00$ mmol $), \mathrm{Hg}(\mathrm{OAc}) 2(3.2 \mathrm{mg}, 0.01 \mathrm{mmol})$, benzaldehyde $(102 \mu \mathrm{L}, 1.00 \mathrm{mmol}),(S, S)-20 \mathbf{a}(37 \mathrm{mg}$, $0.10 \mathrm{mmol})$ in $2 \mathrm{~mL}$ of $\mathrm{CH}_{2} \mathrm{Cl}_{2}$, was obtained $315 \mathrm{mg}(0.86 \mathrm{mmol}, 86 \%)$ of syn-10a as a clear, colorless, viscous oil after column chromatography (3/1 pentane/ether, $\left.\mathrm{SiO}_{2}\right)$. 


\section{Data for syn-10a:}

1슬 $\quad\left(500 \mathrm{MHz}, \mathrm{CDCl}_{3}\right)$

7.37 (m, 4 H, HC(8), HC(9)); 7.30 (m, 1 H, HC(10)); 5.17 (m, 1H, HC(1)); 4.45 (sext, $J=6.1,1 \mathrm{H}, \mathrm{HC}(5)) ; 3.35(\mathrm{~d}, J=2.9,1 \mathrm{H}, \mathrm{HO}) ; 2.96-2.85$ (ABX, 2H, $\left.\mathrm{H}_{2} \mathrm{C}(2)\right) ; 2.73-2.59$ (ABX, 2H, $\left.\mathrm{H}_{2} \mathrm{C}(4)\right) ; 1.24$ (d, $J=6.1,3 \mathrm{H}, \mathrm{H}_{3} \mathrm{C}(6)$ ); 1.07 (s, $\left.21 \mathrm{H}, \mathrm{H}_{3} \mathrm{C}(13), \mathrm{HC}(11)\right)$

${ }^{13} \underline{\mathrm{C} \mathrm{NMR}}: \quad\left(126 \mathrm{MHz}, \mathrm{CDCl}_{3}\right)$

$210.23(\mathrm{C}(3)) ; 142.75(\mathrm{C}(7)) ; 128.47(\mathrm{C}(9)) ; 127.58$ (C(10)); $125.60(\mathrm{C}(8)) ; 69.73$ $(\mathrm{C}(1)) ; 65.26(\mathrm{C}(5)) ; 53.42$ (C(4)); 52.51 (C(2)); 24.00 (C(6)); 18.00 (C(12)); $12.33(\mathrm{C}(11))$

IR: (neat)

3438 (m, br); 2961 (s); 2944 (s); 2893 (s); 2867 (s); 1710 (s); 1463 (m); 1375 (m); 1130 (m); 1092 (m); 1066 (m); 1038 (m); 1014 (m)

MS: $\quad(\mathrm{FI})$

$365.1\left(2, \mathrm{M}^{+}\right) ; 321.1(100) ; 215.0(6)$

Opt. Rot.: $\quad[\alpha]_{\mathrm{D}}^{24}-5.84(\mathrm{c}=1.07, \mathrm{EtOH})$

TLC: $\quad R_{f} 0.14$ (pentane/ether, 3/1) [silica gel, $p$-anisaldehyde]

SFC: $\quad t_{\mathrm{R}} 4.163 \mathrm{~min}(\mathrm{OD}, 150 \mathrm{bar}, 3 \mathrm{~mL} / \mathrm{min}, 5 \% \mathrm{MeOH})$

Analysis: $\quad \mathrm{C}_{21} \mathrm{H}_{36} \mathrm{O}_{3} \mathrm{Si}(364.59)$

Calcd: $\quad$ C, 69.18; H, $9.95 \%$

Found: $\quad$ C, 69.03; H, $10.20 \%$ 


\section{(S)-5-tert-Butyldimethylsilyloxy-3-hexanone (3)}

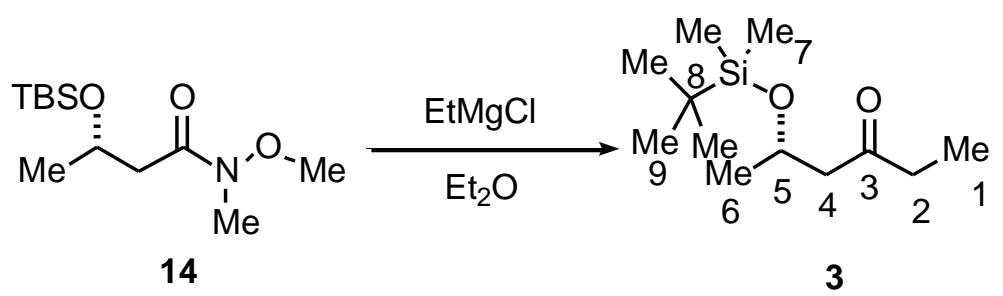

Amide 14 (2.00 g, $7.65 \mathrm{mmol})$ was dissolved in $20 \mathrm{~mL}$ of $\mathrm{Et}_{2} \mathrm{O}$ at $0{ }^{\circ} \mathrm{C}$. To this solution was slowly added a solution of ethylmagnesium chloride $\left(2.0 \mathrm{M}\right.$ in $\mathrm{Et}_{2} \mathrm{O}, 5.75 \mathrm{~mL}, 11.5 \mathrm{mmol}$, 1.5 equiv). The reaction mixture was stirred at $0{ }^{\circ} \mathrm{C}$ for $1 \mathrm{~h}$, then was gradually allowed to warm to $\mathrm{rt}$ and was stirred overnight. The reaction mixture was quenched by slow addition of sat. aq. ammonium chloride solution (ca. $15 \mathrm{~mL}$ ). The layers were separated and the aqueous layer was extracted with $\mathrm{Et}_{2} \mathrm{O}(2 \times 50 \mathrm{~mL})$. The extracts were combined, dried $\left(\mathrm{MgSO}_{4}\right)$, filtered, and concentrated. The crude product was purified by chromatography (hexane/ether, 5/1, $\mathrm{SiO}_{2}$ ) and by distillation to afford $1.60 \mathrm{~g}$ of $\mathbf{3}(6.94 \mathrm{mmol}, 91 \%)$ as clear, colorless liquid.

Data for 3:

bp: $\quad 105^{\circ} \mathrm{C}(0.01 \mathrm{mmHg})$

1슬 $\quad$ (400 MHz, $\left.\mathrm{CDCl}_{3}\right)$

4.28 (sext, $J=6.3,1 \mathrm{H}, \mathrm{HC}(5)) ; 2.65-2.34\left(\mathrm{ABX}, 2 \mathrm{H}, \mathrm{H}_{2} \mathrm{C}(4)\right) ; 2.45$ (q, $J=7.4$, $\left.2 \mathrm{H}, \mathrm{H}_{2} \mathrm{C}(2)\right) ; 1.15$ (d, $\left.J=6.1,3 \mathrm{H}, \mathrm{H}_{3} \mathrm{C}(6)\right) ; 1.02$ (t, $\left.J=7.3,3 \mathrm{H}, \mathrm{H}_{3} \mathrm{C}(1)\right) ; 0.85$ (s, $\left.9 \mathrm{H}, \mathrm{H}_{3} \mathrm{C}(9)\right) ; 0.03$ (d, $J=16.1,6 \mathrm{H}, \mathrm{H}_{3} \mathrm{C}(7)$ )

${ }^{13} \mathrm{C} \mathrm{NMR}: \quad\left(100 \mathrm{MHz}, \mathrm{CDCl}_{3}\right)$

$210.58(\mathrm{C}(3)) ; 65.82(\mathrm{C}(5)) ; 51.92(\mathrm{C}(4)) ; 37.78(\mathrm{C}(2)) ; 25.74(\mathrm{C}(9)) ; 24.09$ $(\mathrm{C}(8)) ; 17.92(\mathrm{C}(6)) ; 7.40(\mathrm{C}(1)) ;-4.56,-5.06(\mathrm{C}(7))$

IR: (neat)

2958 (s); 2932 (s); 2896 (m); 2858 (m); 1718 (s); 1376 (m); 1255 (m); 1135 (m); 1095 (m); 1046 (s)

MS: $\quad(\mathrm{FI})$

$231.1\left(2, \mathrm{M}^{+}\right) ; 173.0(100)$

Opt. Rot.: $\quad[\alpha]_{\mathrm{D}}^{24}+49.0\left(\mathrm{c}=0.92, \mathrm{CHCl}_{3}\right)$ 
TLC: $\quad R_{f} 0.21$ (pentane/ether, 5/1) [silica gel, $\mathrm{KMnO}_{4}$ ]

Analysis: $\quad \mathrm{C}_{12} \mathrm{H}_{26} \mathrm{O}_{2} \mathrm{Si}(230.42)$
Calcd:
C, 62.55;
$\mathrm{H}, 11.37 \%$
Found:
C, 62.57;
$\mathrm{H}, 11.45 \%$

\section{(2Z,5S)- 5-tert-Butyldimethylsilyloxy-3-trimethylsilyloxy-2-hexene $\quad((Z)-18) \quad$ (Using DBU/TMSCl)}

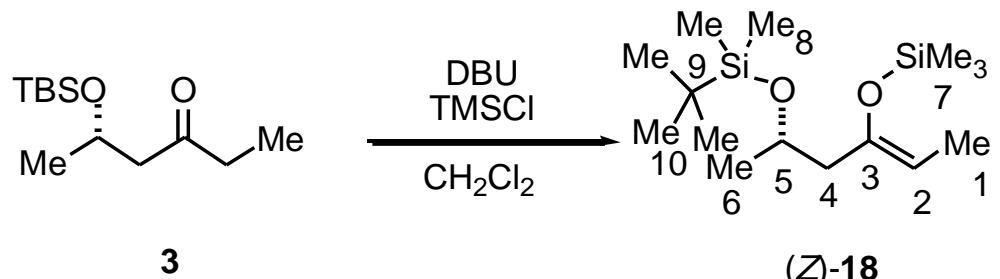

In a 50-mL, three-neck flask equipped with a stopper and a reflux condenser, DBU (1.30 $\mathrm{mL}, 8.68 \mathrm{mmol}, 2$ equiv) was dissolved in $20 \mathrm{~mL}$ of $\mathrm{CH}_{2} \mathrm{Cl}_{2}$. To this solution were added TMSCl (1.10 mL, $8.68 \mathrm{mmol}, 2$ equiv) and ketone 3 (1.00 g, $4.34 \mathrm{mmol})$ and the mixture was heated to reflux for $4 \mathrm{~h}$. The reaction mixture was cooled to $\mathrm{rt}$ and was quenched with $20 \mathrm{~mL}$ of cold, sat. aq. $\mathrm{NaHCO}_{3}$ solution. The product was extracted with $50 \mathrm{~mL}$ of hexane and the hexane was washed with $25 \mathrm{~mL}$ of brine. The extracts were dried $\left(\mathrm{Na}_{2} \mathrm{SO}_{4}\right)$, filtered, and concentrated. The crude product was purified by chromatography (hexane/ $\mathrm{CH}_{2} \mathrm{Cl}_{2}, 5 / 1$ ) and Kugelrohr distillation to give $820 \mathrm{mg}$ of $(Z)-\mathbf{1 8}(2.71 \mathrm{mmol}, 62 \%)$ as clear colorless oil.

Data for $(Z)-\mathbf{1 8}$ :

bp: $\quad 125^{\circ} \mathrm{C}(0.5 \mathrm{mmHg}, \mathrm{ABT})$

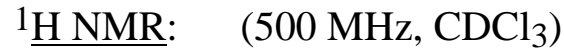

4.54 (q, $J=6.6,1 \mathrm{H}, \mathrm{HC}(2)) ; 3.99$ (sext, $J=6.2,1 \mathrm{H}, \mathrm{HC}(4)$ ); 2.21-1.97 (ABX, 2 $\left.\mathrm{H}, \mathrm{H}_{2} \mathrm{C}(4)\right) ; 1.48$ (d, $\left.J=6.7,3 \mathrm{H}, \mathrm{H}_{3} \mathrm{C}(1)\right) ; 1.13$ (d, $\left.J=6.0,3 \mathrm{H}, \mathrm{H}_{3} \mathrm{C}(6)\right) ; 0.87$ (s, $\left.9 \mathrm{H}, \mathrm{H}_{3} \mathrm{C}(10)\right) ; 0.18\left(\mathrm{~s}, 9 \mathrm{H}, \mathrm{H}_{3} \mathrm{C}(7)\right) ; 0.03\left(\mathrm{~d},, J=6.2,6 \mathrm{H}, \mathrm{H}_{3} \mathrm{C}(8)\right)$

${ }^{13} \mathrm{C} \mathrm{NMR}: \quad\left(126 \mathrm{MHz}, \mathrm{CDCl}_{3}\right)$

148.47 (C(3)); 104.68 (C(2)); 66.44 (C(5)); 47.48 (C(4); 25.84 (C(10)); 23.54 $(\mathrm{C}(9)) ; 18.12(\mathrm{C}(6)) ; 10.82(\mathrm{C}(1)) ; 0.64(\mathrm{C}(7)) ;-4.71,-4.86(\mathrm{C}(8))$ 
IR: (neat)

2958 (s); 2929 (m); 2899 (m); 2858 (m); 1680 (m); 1473 (m); 1377 (m); 1323

(m); 1254 (s); 1194 (m); 1128 (m); 1093 (m); 1070 (m); 1030 (m)

$\underline{\mathrm{MS}}: \quad(\mathrm{FI})$

$302.1\left(100, \mathrm{M}^{+}\right)$

Opt. Rot.: $\quad[\alpha]_{\mathrm{D}}^{24}+4.61\left(\mathrm{c}=0.97, \mathrm{CHCl}_{3}\right)$

TLC: $\quad R_{f} 0.20$ (hexane/ $\left.\mathrm{CH}_{2} \mathrm{Cl}_{2}, 5 / 1\right)$ [silica gel, $\mathrm{KMnO}_{4}$ ]

Analysis: $\quad \mathrm{C}_{15} \mathrm{H}_{34} \mathrm{O}_{2} \mathrm{Si}_{2}(302.60)$

Calcd: $\quad$ C, $59.54 ; \quad H, 11.33 \%$

Found: $\quad$ C, $59.67 ; \quad H, 11.61 \%$

\section{(S)-5-Triisopropylsilyloxy-3-hexanone (4)}

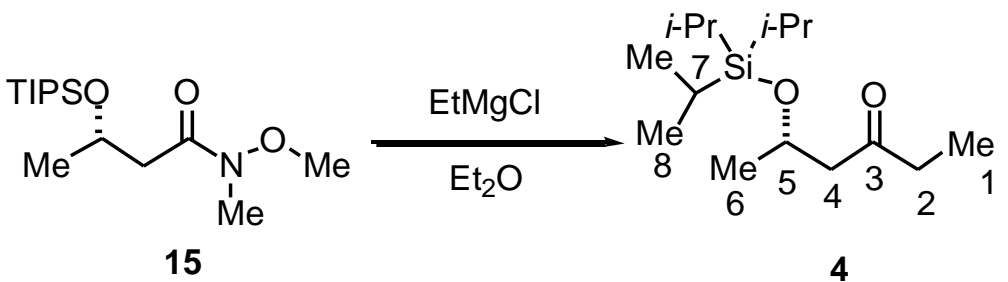

Amide 15 (3.34 g, $11.0 \mathrm{mmol})$ was dissolved in $20 \mathrm{~mL}$ of $\mathrm{Et}_{2} \mathrm{O}$ at $0{ }^{\circ} \mathrm{C}$. To this solution was slowly added a solution of ethylmagnesium chloride $\left(2.0 \mathrm{M}\right.$ in $\mathrm{Et}_{2} \mathrm{O}, 8.25 \mathrm{~mL}, 16.5 \mathrm{mmol}$, 1.5 equiv). The reaction mixture was stirred at $0{ }^{\circ} \mathrm{C}$ for $1 \mathrm{~h}$, then was gradually allowed to warm to $\mathrm{rt}$ and was stirred overnight. The reaction mixture was quenched by slow addition of sat. aq. ammonium chloride solution (ca. $20 \mathrm{~mL}$ ). The layers were separated and the aqueous layer was extracted with $\mathrm{Et}_{2} \mathrm{O}(2 \times 50 \mathrm{~mL})$. The extracts were combined, dried $\left(\mathrm{MgSO}_{4}\right)$, filtered, and concentrated. The crude product was purified by chromatography (hexane/ether, $5 / 1, \mathrm{SiO}_{2}$ ) and by distillation to afford $2.60 \mathrm{~g}$ of $4(9.54 \mathrm{mmol}, 87 \%)$ as clear, colorless liquid.

\section{Data for 4:}

bp: $\quad 104{ }^{\circ} \mathrm{C}(0.1 \mathrm{mmHg})$ 
${ }^{1} \underline{\mathrm{H} \text { NMR}}: \quad\left(500 \mathrm{MHz}, \mathrm{CDCl}_{3}\right)$

4.42 (sext, $J=6.1,1 \mathrm{H}, \mathrm{HC}(5)) ; 2.69-2.44\left(\mathrm{ABX}, 2 \mathrm{H}, \mathrm{H}_{2} \mathrm{C}(4)\right) ; 2.46$ (q, $J=7.1$, $\left.2 \mathrm{H}, \mathrm{H}_{2} \mathrm{C}(2)\right) ; 1.20\left(\mathrm{~d}, J=5.9,3 \mathrm{H}, \mathrm{H}_{3} \mathrm{C}(6)\right) ; 1.07-1.02\left(\mathrm{~m}, 24 \mathrm{H}, \mathrm{H}_{3} \mathrm{C}(1)\right.$, $\left.\mathrm{H}_{3} \mathrm{C}(8), \mathrm{HC}(7)\right)$

${ }^{13} \mathrm{C}$ NMR: $\quad\left(126 \mathrm{MHz}, \mathrm{CDCl}_{3}\right)$

$210.45(\mathrm{C}(3)) ; 65.57(\mathrm{C}(5)) ; 52.36(\mathrm{C}(4)) ; 37.49$ (C(2)); $24.15(\mathrm{C}(6)) ; 18.05$ $(\mathrm{C}(8)) ; 12.34(\mathrm{C}(7)) ; 7.45(\mathrm{C}(1))$

IR: (neat)

2964 (s); 2944 (s); 2894 (m); 2868 (s); 1718 (s); 1463 (m); 1412 (m); 1375 (m); 1134 (m); 1098 (m); $1044(\mathrm{~m})$

MS: $\quad(\mathrm{FI})$

$273.2\left(2, \mathrm{M}^{+}\right) ; 231.2(5) ; 230.2(19) ; 229.2(100)$

Opt. Rot.: $\quad[\alpha]_{\mathrm{D}}^{24}+12.8\left(\mathrm{c}=0.65, \mathrm{CHCl}_{3}\right)$

TLC: $\quad R_{f} 0.25$ (hexane/ether, 5/1) [silica gel, $\mathrm{KMnO}_{4}$ ]

Analysis: $\quad \mathrm{C}_{15} \mathrm{H}_{32} \mathrm{O}_{2} \mathrm{Si}(272.50)$

$\begin{array}{lll}\text { Calcd: } & \text { C }, 66.11 ; & \text { H }, 11.84 \% \\ \text { Found: } & \text { C, 66.22; } & \text { H, } 11.93 \%\end{array}$

(2Z,5S)- 5-Triisopropylsilyloxy-3-trimethylsilyloxy-2-hexene ((Z)-19)

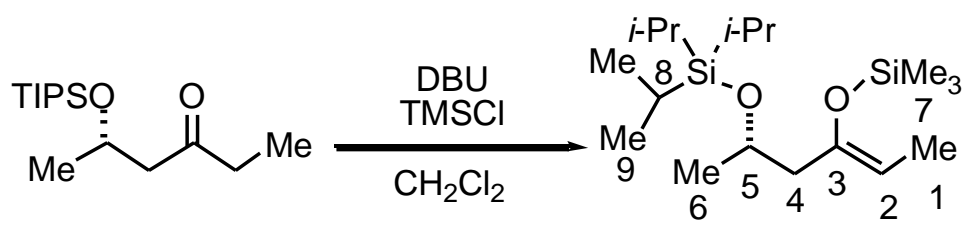

4

(Z)-19

In a 50-mL, three-neck flask equipped with a stopper and a reflux condenser, DBU (0.89 $\mathrm{mL}, 6.06 \mathrm{mmol}, 1.5$ equiv) was dissolved in $15 \mathrm{~mL}$ of $\mathrm{CH}_{2} \mathrm{Cl}_{2}$. To this solution were added TMSCl (0.77 mL, $6.06 \mathrm{mmol}, 1.5$ equiv) and ketone 4 (1.10 g, $4.04 \mathrm{mmol})$ and the reaction mixture was heated to reflux for $4 \mathrm{~h}$. The reaction mixture was cooled to $\mathrm{rt}$ and was quenched with $20 \mathrm{~mL}$ of cold, sat. aq. $\mathrm{NaHCO}_{3}$ solution. The product was extracted with $50 \mathrm{~mL}$ of hexane 
and the hexane was washed with $25 \mathrm{~mL}$ of brine. The extracts were dried $\left(\mathrm{Na}_{2} \mathrm{SO}_{4}\right)$, filtered, and concentrated. The crude product was purified by chromatography (hexane/ $\mathrm{CH}_{2} \mathrm{Cl}_{2}, 8 / 1$ ) and Kugelrohr distillation to give $800 \mathrm{mg}$ of (Z)-19 (2.32 mmol, 57\%) as clear, colorless oil.

Data for (Z)-19:

bp: $\quad 125^{\circ} \mathrm{C}(0.05 \mathrm{mmHg}, \mathrm{ABT})$

1슬 $: \quad\left(400 \mathrm{MHz}, \mathrm{CDCl}_{3}\right)$

4.55 (q, $J=6.8,1 \mathrm{H}, \mathrm{HC}(2)) ; 4.10$ (sextd, $J=5.6,2.7,1 \mathrm{H}, \mathrm{HC}(5)) ; 2.33-1.97$ $\left(\mathrm{ABX}, 2 \mathrm{H}, \mathrm{H}_{2} \mathrm{C}(4)\right) ; 1.48\left(\mathrm{~d}, J=6.8,3 \mathrm{H}, \mathrm{H}_{3} \mathrm{C}(1)\right) ; 1.17(\mathrm{~d}, J=5.9,3 \mathrm{H}$, $\left.\mathrm{H}_{3} \mathrm{C}(6)\right) ; 1.16-1.05\left(\mathrm{~m}, 21 \mathrm{H}, \mathrm{H}_{3} \mathrm{C}(9), \mathrm{HC}(8)\right) ; 0.18$ (s, $\left.9 \mathrm{H}, \mathrm{H}_{3} \mathrm{C}(7)\right)$

${ }^{13} \underline{\mathrm{C} \mathrm{NMR}}: \quad\left(126 \mathrm{MHz}, \mathrm{CDCl}_{3}\right)$

148.71 (C(3)); $104.67(\mathrm{C}(2)) ; 66.74$ (C(5)); 47.80 (C(4); 23.56 (C(6)); 18.09 (C(9)); 12.44 (C(8)); 10.92 (C(1)); 0.64 (C(7))

IR: (neat)

2961 (s); 2944 (s); 2895 (m); 2867 (s); 1678 (m); 1464 (m); 1377 (m); 1323 (m); 1253 (s); 1193 (m); 1128 (m); 1096 (m); 1069 (m); 1028 (m); 1004 (m)

MS: $\quad(\mathrm{FI})$

346.3 (12); 345.3 (33); $344.3\left(100, \mathrm{M}^{+}\right) ; 263.6(3)$

Opt. Rot.: $\quad[\alpha]_{\mathrm{D}}^{24}+6.57\left(\mathrm{c}=0.48, \mathrm{CHCl}_{3}\right)$

TLC: $\quad R_{f} 0.25$ (hexane/ $\left.\mathrm{CH}_{2} \mathrm{Cl}_{2}, 8 / 1\right)$ [silica gel, $\mathrm{KMnO}_{4}$ ]

Analysis: $\quad \mathrm{C}_{18} \mathrm{H}_{40} \mathrm{O}_{2} \mathrm{Si}_{2}(344.68)$

Calcd: $\quad$ C, 62.72; H, $11.70 \%$

Found: $\quad$ C, $62.53 ; \quad H, 12.00 \%$ 


\section{(2E,5S)- 5-Triisopropylsilyloxy-3-trimethylsilyloxy-2-hexene $((E)-19)$}

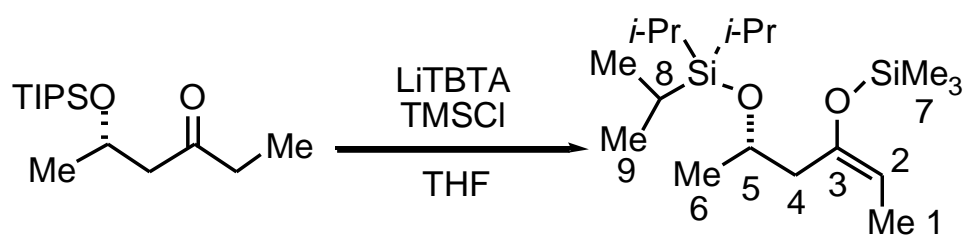

4

(E)-19

In a $25-\mathrm{mL}$, two-neck flask equipped with a stopper and a septum tert-butyltritylamine (TBTA) (946 mg, $3.00 \mathrm{mmol}, 1.5$ equiv) was dissolved in $10 \mathrm{~mL}$ of THF at $0{ }^{\circ} \mathrm{C}$. To this solution was added dropwise a solution of $n$-BuLi (1.54 M in hexane, $1.82 \mathrm{~mL}, 2.80 \mathrm{mmol}, 1.4$ equiv) and the solution was stirred for $10 \mathrm{~min}$. The mixture was cooled to $-78{ }^{\circ} \mathrm{C}$, whereupon TMSCl (0.381 mL, $3.00 \mathrm{mmol}, 1.5$ equiv) and 4 (0.55 g, $2.00 \mathrm{mmol})$ were added. The reaction mixture was stirred for $30 \mathrm{~min}$ and then was gradually warmed to $0{ }^{\circ} \mathrm{C}$ over $2 \mathrm{~h}$. The reaction mixture was quenched with $10 \mathrm{~mL}$ of cold, sat. aq. $\mathrm{NaHCO}_{3}$ solution. The product was extracted in $50 \mathrm{~mL}$ of pentane and the pentane was washed with $25 \mathrm{~mL}$ of brine. The extracts were dried $\left(\mathrm{Na}_{2} \mathrm{SO}_{4}\right)$, filtered, and concentrated. The residue was redissolved in pentane and was cooled to $-15{ }^{\circ} \mathrm{C}$ to recover TBTA. The crude product was purified by chromatography (hexane $/ \mathrm{CH}_{2} \mathrm{Cl}_{2}, 8 / 1, \mathrm{SiO}_{2}$ ) and Kugelrohr distillation to give $475 \mathrm{mg}$ of $(E)-19$ (1.38 mmol, $69 \%$ ) as clear, colorless oil.

Data for $(E)-19$ :

bp: $\quad 125^{\circ} \mathrm{C}(0.05 \mathrm{mmHg}, \mathrm{ABT})$

${ }^{1} \underline{\mathrm{H} \text { NMR: }} \quad\left(400 \mathrm{MHz}, \mathrm{CDCl}_{3}\right)$

$4.67(\mathrm{q}, J=6.8,1 \mathrm{H}, \mathrm{HC}(2)) ; 4.14(\mathrm{~m}, 1 \mathrm{H}, \mathrm{HC}(5)) ; 2.32-2.23(\mathrm{ABX}, 2 \mathrm{H}$, $\left.\mathrm{H}_{2} \mathrm{C}(4)\right) ; 1.55\left(\mathrm{dd}, J=6.8,0.5,3 \mathrm{H}, \mathrm{H}_{3} \mathrm{C}(1)\right) ; 1.16\left(\mathrm{~d}, J=6.1,3 \mathrm{H}, \mathrm{H}_{3} \mathrm{C}(6)\right) ; 1.07$ (s, $\left.21 \mathrm{H}, \mathrm{H}_{3} \mathrm{C}(9), \mathrm{HC}(8)\right) ; 0.15$ (s, $9 \mathrm{H}, \mathrm{H}_{3} \mathrm{C}(7)$ )

${ }^{13}$ C NMR: $\quad\left(126 \mathrm{MHz}, \mathrm{CDCl}_{3}\right)$

$149.61(\mathrm{C}(3)) ; 102.60(\mathrm{C}(2)) ; 67.01$ (C(5)); 42.03 (C(4); 23.15 (C(6)); 18.11 $(\mathrm{C}(9)) ; 12.40(\mathrm{C}(8)) ; 12.06(\mathrm{C}(1)) ; 0.23(\mathrm{C}(7))$ 
IR: (neat)

2962 (m); 2945 (m); 2895 (m); 2868 (m); $1670(\mathrm{w}) ; 1464(\mathrm{~m}) ; 1379(\mathrm{w}) ; 1254$

(m); 1198 (m); $1124(\mathrm{~m}) ; 1107$ (m); 1088 (m); 1005 (m)

MS: $\quad(\mathrm{FI})$

347.2 (2); 346.2 (11); 345.2 (30); 344.2 (100, $\left.\mathrm{M}^{+}\right) ; 343.7$ (2); 264.2 (2); 263.8 (3);

$263.6(5) ; 263.3(3)$

Opt. Rot.: $\quad[\alpha]_{\mathrm{D}}^{24}+6.64\left(\mathrm{c}=0.39, \mathrm{CHCl}_{3}\right)$

TLC: $\quad R_{f}=0.26\left(\right.$ hexane/ $\left.\mathrm{CH}_{2} \mathrm{Cl}_{2}, 8 / 1\right)$ [silica gel, $\mathrm{KMnO}_{4}$ ]

Analysis: $\quad \mathrm{C}_{118} \mathrm{H}_{40} \mathrm{O}_{2} \mathrm{Si}_{2}(344.68)$

Calcd: $\quad$ C, 62.72; $\quad$ H $11.70 \%$

Found: $\quad$ C, $62.38 ; \quad H, 11.94 \%$

\section{General Procedure II: (2Z,5S)- 5-tert-Butyldimethylsilyloxy-3-trichlorosilyloxy-2-hexene} $((Z)-7)$

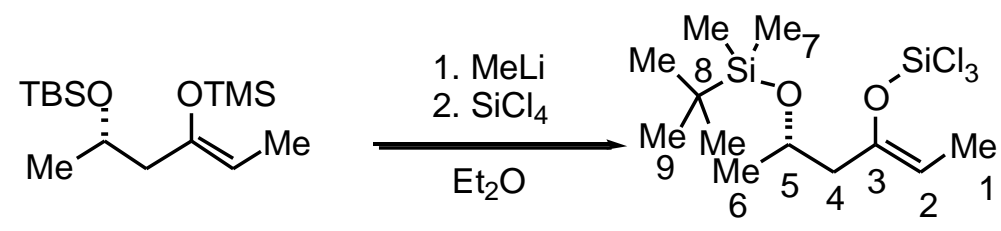

$(Z)-18$

$(Z)-7$

To a solution of silyl enol ether (Z)-18 (972 mg, $3.21 \mathrm{mmol})$ in $3 \mathrm{~mL}$ of $\mathrm{Et}_{2} \mathrm{O}$ at $0{ }^{\circ} \mathrm{C}$ was slowly added a solution of $\mathrm{MeLi}\left(1.5 \mathrm{M}\right.$ in $\mathrm{Et}_{2} \mathrm{O}, 3.30 \mathrm{~mL}, 4.82 \mathrm{mmol}, 1.5$ equiv). The reaction mixture was stirred for $3 \mathrm{~h}$ at $\mathrm{rt}$, then was cooled to $-78{ }^{\circ} \mathrm{C}$. The reaction mixture was then transferred via cannula to a cold solution of silicon tetrachloride (3.68 mL, $32.1 \mathrm{mmol}, 10$ equiv) in $2 \mathrm{~mL}$ of $\mathrm{Et}_{2} \mathrm{O}$. The reaction mixture was stirred at $-78{ }^{\circ} \mathrm{C}$ for $1 \mathrm{~h}$ and was gradually allowed to warm to rt. The precipitate was allowed to settle to the bottom of the flask, the supernatant was transferred to another flask and was concentrated in vacuo. The product was purified by Kugelrohr distillation to afford $764 \mathrm{mg}(2.10 \mathrm{mmol}, 65 \%)$ of $(Z)-7$ as a clear, colorless oil. 
Data for $(Z)-7$ :

bp: $\quad 150{ }^{\circ} \mathrm{C}(1.0 \mathrm{mmHg}, \mathrm{ABT})$

1ㅁN $\underline{\mathrm{HMR}}: \quad\left(\mathrm{CDCl}_{3}, 500 \mathrm{MHz}\right)$

4.83 (q, $J=6.9,1 \mathrm{H}, \mathrm{HC}(2)) ; 4.03$ (sext, $J=7.3,1 \mathrm{H}, \mathrm{HC}(5)$ ); 2.33-2.18 (ABX, 2 $\left.\mathrm{H}, \mathrm{H}_{2} \mathrm{C}(4)\right) ; 1.58$ (d, $\left.J=6.8,3 \mathrm{H}, \mathrm{H}_{3} \mathrm{C}(1)\right) ; 1.15$ (d, $\left.J=6.2,3 \mathrm{H}, \mathrm{H}_{3} \mathrm{C}(6)\right) ; 0.87$ (s, $\left.9 \mathrm{H}, \mathrm{H}_{3} \mathrm{C}(9)\right) ; 0.03\left(\mathrm{~d}, J=10.5,6 \mathrm{H}, \mathrm{H}_{3} \mathrm{C}(7)\right)$

\section{(2Z,5S)- 5-Triisopropylsilyloxy-3-trichlorosilyloxy-2-hexene ((Z)-8)}

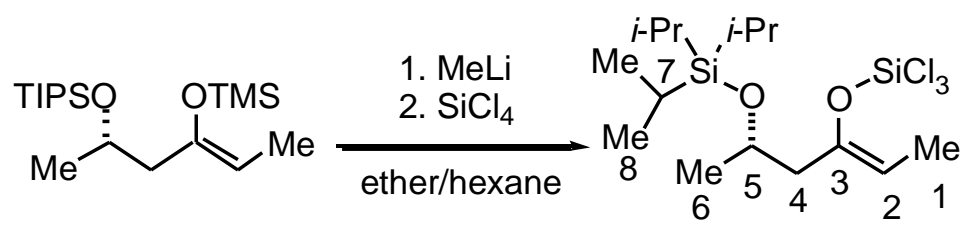

$(Z)-19$

$(Z)-8$

Following the General Procedure II from (Z)-19 (1.60 g, $4.64 \mathrm{mmol})$, MeLi (1.5 M in $\mathrm{Et}_{2} \mathrm{O}, 4.64 \mathrm{~mL}, 6.96 \mathrm{mmol}, 1.5$ equiv) and $\mathrm{SiCl}_{4}(5.32 \mathrm{~mL}, 46.4 \mathrm{mmol}, 10$ equiv) in $10 \mathrm{~mL}$ of hexane was obtained $1.13 \mathrm{~g}(2.78 \mathrm{mmol}, 60 \%)$ of $(Z)-8$ as a clear, colorless oil.

Data for $(Z)-8$ :

bp: $\quad 175^{\circ} \mathrm{C}(1.0 \mathrm{mmHg}, \mathrm{ABT})$

${ }^{1} \underline{\mathrm{H} N M R}: \quad\left(500 \mathrm{MHz}, \mathrm{CDCl}_{3}\right)$

$4.84(\mathrm{q}, J=6.8,1 \mathrm{H}, \mathrm{HC}(2)) ; 4.16$ (sext, $J=7.1,1 \mathrm{H}, \mathrm{HC}(5))$; 2.47-2.17 (ABX, 2 $\left.\mathrm{H}, \mathrm{H}_{2} \mathrm{C}(4)\right) ; 1.59$ (dt, $\left.J=6.8,1.0,3 \mathrm{H}, \mathrm{H}_{3} \mathrm{C}(3)\right) ; 1.20$ (d, $\left.J=5.9,3 \mathrm{H}, \mathrm{H}_{3} \mathrm{C}(6)\right)$; $1.13-1.01\left(\mathrm{~m}, 21 \mathrm{H}, \mathrm{H}_{3} \mathrm{C}(8), \mathrm{HC}(7)\right)$ 


\section{(2E,5S)- 5-Triisopropylsilyloxy-3-trichlorosilyloxy-2-hexene $((E)-8)$}

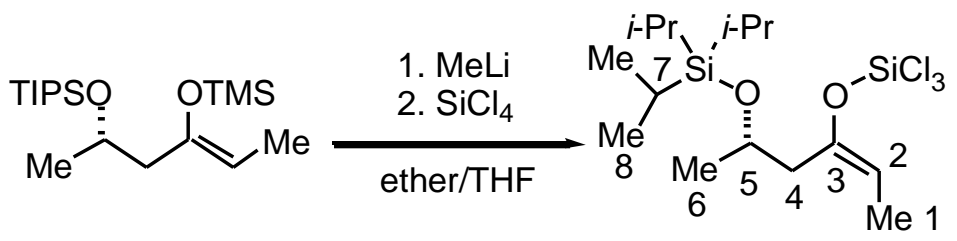

$(E)-19$

(E)-8

Following General Procedure II from $(E)-19$ (678 mg, $1.97 \mathrm{mmol})$, MeLi (1.5 M in Et $2 \mathrm{O}$, $1.57 \mathrm{~mL}, 2.36 \mathrm{mmol}, 1.2$ equiv) and $\mathrm{SiCl}_{4}(2.26 \mathrm{~mL}, 19.7 \mathrm{mmol}, 1.0$ equiv) in $5 \mathrm{~mL}$ of ether, was obtained $180 \mathrm{mg}(0.44 \mathrm{mmol}, 23 \%)$ of $(E)-8$ as a clear, colorless oil.

Data for $(E)-8$ :

bp: $\quad 150{ }^{\circ} \mathrm{C}(0.5 \mathrm{mmHg}, \mathrm{ABT})$

1슬 $: \quad\left(500 \mathrm{MHz}, \mathrm{CDCl}_{3}\right)$

$5.17(\mathrm{q}, J=7.1,1 \mathrm{H}, \mathrm{HC}(2)) ; 4.17(\mathrm{~m}, 1 \mathrm{H}, \mathrm{HC}(5)) ; 2.56-2.31(\mathrm{ABX}, 2 \mathrm{H}$, $\left.\mathrm{H}_{2} \mathrm{C}(4)\right) ; 1.62$ (d, $\left.J=7.1,3 \mathrm{H}, \mathrm{H}_{3} \mathrm{C}(3)\right) ; 1.20$ (d, $\left.J=6.1,3 \mathrm{H}, \mathrm{H}_{3} \mathrm{C}(6)\right) ; 1.09-$ $1.02\left(\mathrm{~m}, 21 \mathrm{H}, \mathrm{H}_{3} \mathrm{C}(8), \mathrm{HC}(7)\right)$

General Procedure III: $(1 R, 2 R, 5 S)$-5-tert-Butyldimethylsilyloxy-2-methyl-1-hydroxy-1phenyl-3-hexanone (syn,anti-11a)<smiles>CC=C(CC(C)O[SbH3])O[GeH3]</smiles>

(Z)-7
$\mathrm{PhCHO}$

a

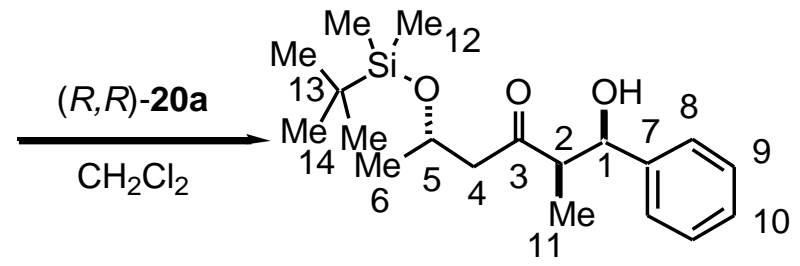

(syn,anti)-11a

Trichlorosilyl enol ether (Z)-7 (364 mg, $1.00 \mathrm{mmol})$ was added to a solution of $(R, R)-\mathbf{2 0 a}$ (37 mg, $0.10 \mathrm{mmol}, 0.10$ equiv) in $1.00 \mathrm{~mL}$ of $\mathrm{CH}_{2} \mathrm{Cl}_{2}$. The reaction mixture was cooled to -78 ${ }^{\circ} \mathrm{C}$ and $\mathrm{PhCHO}(102 \mu \mathrm{L}, 1.00 \mathrm{mmol}, 1$ equiv) was added by syringe. After stirring for $6 \mathrm{~h}$, the reaction mixture was quenched by pouring it into a vigorously stirring solution of cold, sat. aq. 
$\mathrm{NaHCO}_{3}$ solution $(15 \mathrm{~mL})$. The resulting slurry was stirred for $3 \mathrm{~h}$ and was filtered through Celite. The layers were separated, and the aqueous layer was extracted with $20 \mathrm{~mL}$ of $\mathrm{CH}_{2} \mathrm{Cl}_{2}$. The organic phases were combined, washed with $5 \mathrm{~mL}$ of brine and were dried $\left(\mathrm{Na}_{2} \mathrm{SO}_{4}\right)$, filtered, and concentrated. The crude product was purified by column chromatography (pentane/ether, 3/1, $\left.\mathrm{SiO}_{2}\right)$ to afford $200 \mathrm{mg}(0.59 \mathrm{mmol}, 59 \%)$ of $\mathbf{1 1 a}$ as a clear, viscous oil.

Data for (syn,anti)-11a:

${ }^{1} \underline{\mathrm{H} \mathrm{NMR}}: \quad\left(500 \mathrm{MHz}, \mathrm{CDCl}_{3}\right)$

7.36-7.30 (m, $4 \mathrm{H}, \mathrm{HC}(8), \mathrm{HC}(9))$; 7.25 (m, $1 \mathrm{H}, \mathrm{HC}(10))$; 5.15 (t, J = 2.9, $1 \mathrm{H}$, $\mathrm{HC}(1)) ; 4.32$ (sext, $J=6.6,1 \mathrm{H}, \mathrm{HC}(5)) ; 3.16$ (d, $J=2.6,1 \mathrm{H}, \mathrm{HO}) ; 2.81$ (qd, $J=$ 7.3, 3.1, $1 \mathrm{H}, \mathrm{HC}(2)) ; 2.72-2.43\left(\mathrm{ABX}, 2 \mathrm{H}, \mathrm{H}_{2} \mathrm{C}(4)\right) ; 1.16(\mathrm{~d}, J=6.1,3 \mathrm{H}$, $\left.\mathrm{H}_{3} \mathrm{C}(6)\right) ; 1.04$ (d, J= 7.3, $3 \mathrm{H}, \mathrm{H}_{3} \mathrm{C}(11)$ ); 0.87 (s, 9H, $\left.\mathrm{H}_{3} \mathrm{C}(14)\right) ; 0.06$ (d, J = 11.2, $\left.6 \mathrm{H}, \mathrm{H}_{3} \mathrm{C}(12)\right)$

${ }^{13}$ C NMR: $\quad\left(126 \mathrm{MHz}, \mathrm{CDCl}_{3}\right)$

$214.67(\mathrm{C}(3)) ; 141.62(\mathrm{C}(7)) ; 128.22(\mathrm{C}(9)) ; 127.23(\mathrm{C}(10)) ; 125.82(\mathrm{C}(8)) ; 72.33$ $(\mathrm{C}(5)) ; 65.43(\mathrm{C}(1)) ; 52.53(\mathrm{C}(2)) ; 51.30$ (C(4)); 25.77 (C(14)); 23.94 (C(6)); $17.91(\mathrm{C}(13)) ; 9.22(\mathrm{C}(11)) ;-4.58,-4.92(\mathrm{C}(12))$

IR: (neat) 3458 (br, m); 3064 (w); 3032 (w); 2956 (m); 2931 (m); 2895 (m); 2858 (m); 1707 (m); 1454 (m); 1375 (m); 1255 (m); 1134 (m); 1095 (m); 1005 (m)

$\underline{\mathrm{MS}}: \quad(\mathrm{FI})$

$336.2\left(\mathrm{M}^{+}, 7\right) ; 279.1(100) ; 230.2(5) ; 173.1(3) ; 107.2(5)$

Opt. Rot.: $\quad[\alpha]_{\mathrm{D}}^{24}-5.55(\mathrm{c}=0.63, \mathrm{EtOH})$

TLC: $\quad R_{f} 0.20$ (pentane/ether, 5/1) [silica gel, $p$-anisaldehyde]

SFC: $\quad t_{\mathrm{R}} 2.441 \mathrm{~min}(\mathrm{OD}, 150 \mathrm{bar}, 3 \mathrm{~mL} / \mathrm{min}, 5 \% \mathrm{MeOH})$

Analysis: $\quad \mathrm{C}_{19} \mathrm{H}_{32} \mathrm{O}_{3} \mathrm{Si}(336.54)$

Calcd: $\quad$ C, $67.81 ; \quad H, 9.58 \%$

Found: $\quad$ C, $67.61 ; \quad H, 9.59 \%$ 


\section{(1S,2S,5S)-5-tert-Butyldimethylsilyloxy-2-methyl-1-hydroxy-1-phenyl-3-hexanone (syn,syn-}

11a)

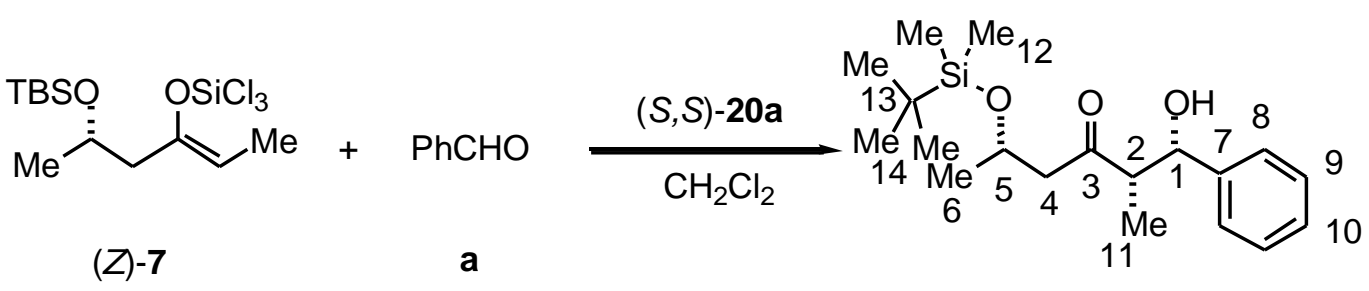

(syn,syn)-11a

Following General Procedure III from (Z)-7 (364 mg, $1.00 \mathrm{mmol}), \mathrm{PhCHO}(102 \mu \mathrm{L}, 1.00$ mmol, 1.0 equiv) and $(S, S)-\mathbf{2 0 a}\left(37 \mathrm{mg}, 0.10 \mathrm{mmol}, 0.1\right.$ equiv) in $1 \mathrm{~mL}$ of $\mathrm{CH}_{2} \mathrm{Cl}_{2}$, was obtained $203 \mathrm{mg}(0.60 \mathrm{mmol}, 60 \%)$ of 11a as a clear, viscous oil after column chromatography (pentane/ether, $3 / 1, \mathrm{SiO}_{2}$ ). The product was further purified by recrystallization from pentane.

Data for $(s y n, s y n)-11 a:$

mp: $\quad 46-48^{\circ} \mathrm{C}$ (pentane)

${ }^{1}$ H NMR: $\quad\left(500 \mathrm{MHz}, \mathrm{CDCl}_{3}\right)$

7.35-7.31 (m, 4 H, HC(8), HC(9)); 7.27 - 7.25 (m, 1 H, HC(10)); 5.09 (t, $J=3.4$, $1 \mathrm{H}, \mathrm{HC}(1)) ; 4.32$ (sext, $J=6.1,1 \mathrm{H}, \mathrm{HC}(5)) ; 3.19$ (d, $J=2.5,1 \mathrm{H}, \mathrm{HO}) ; 2.81$ (qd, $J=7.3,3.7,1 \mathrm{H}, \mathrm{HC}(2))$; 2.76-2.34 (ABX, $\left.2 \mathrm{H}, \mathrm{H}_{2} \mathrm{C}(4)\right) ; 1.12$ (d, $J=6.1,3 \mathrm{H}$, $\left.\mathrm{H}_{3} \mathrm{C}(6)\right) ; 1.05$ (d, $\left.J=7.1,3 \mathrm{H}, \mathrm{H}_{3} \mathrm{C}(11)\right) ; 0.85$ (s, 9H, $\left.\mathrm{H}_{3} \mathrm{C}(14)\right) ; 0.05$ (d, $J=16.8$, $\left.6 \mathrm{H}, \mathrm{H}_{3} \mathrm{C}(12)\right)$

${ }^{13} \underline{\mathrm{C} \mathrm{NMR}}: \quad\left(126 \mathrm{MHz}, \mathrm{CDCl}_{3}\right)$

214.18 (C(3)); $141.70(\mathrm{C}(7)) ; 128.24$ (C(9)); 127.29 (C(10)); 125.89 (C(8)); 72.77 $(\mathrm{C}(5)) ; 65.92(\mathrm{C}(1)) ; 53.26(\mathrm{C}(2)) ; 51.45(\mathrm{C}(4)) ; 25.73(\mathrm{C}(14)) ; 23.81(\mathrm{C}(6))$; 17.89 (C(13)); $9.36(\mathrm{C}(11)) ;-4.65,-4.92(\mathrm{C}(12))$

IR: (neat)

3487 (br, m); 2956 (m); 2931 (m); 2885 (m); 2858 (m); 1709 (m); 1454 (m); 1408 (w); $1375(\mathrm{~m}) ; 1254(\mathrm{~m}) ; 1154(\mathrm{~m}) ; 1093$ (m)

MS: $\quad(\mathrm{FI})$

$336.2\left(\mathrm{M}^{+}, 8\right) ; 279.2(100) ; 230.2(7) ; 173.1(10) ; 106.1(7)$ 
Opt. Rot.: $\quad[\alpha]_{\mathrm{D}}^{24}+50.3(\mathrm{c}=0.29, \mathrm{EtOH})$

TLC: $\quad R_{f} 0.20$ (pentane/ether, 5/1) [silica gel, $p$-anisaldehyde]

SFC: $\quad t_{\mathrm{R}} 3.030 \mathrm{~min}(\mathrm{OD}, 150 \mathrm{bar}, 3 \mathrm{~mL} / \mathrm{min}, 5 \% \mathrm{MeOH})$

Analysis: $\quad \mathrm{C}_{19} \mathrm{H}_{32} \mathrm{O}_{3} \mathrm{Si}(336.54)$

$\begin{array}{lll}\text { Calcd: } & \text { C }, 67.81 ; & \text { H, } 9.58 \% \\ \text { Found: } & \text { C }, 67.78 ; & \text { H, } 9.61 \%\end{array}$

$(1 R, 2 R, 5 S)-5-T r i i s o p r o p y l s i l y l o x y-2-m e t h y l-1-h y d r o x y-1-p h e n y l-3-h e x a n o n e ~(s y n, a n t i-12 a)$<smiles>CCOC(C)CC(C)OC</smiles>

$(Z)-8$<smiles>O=CP</smiles>

a

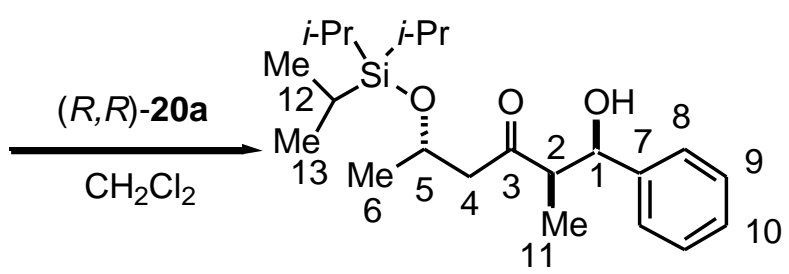

(syn,anti)-12a

Following General Procedure III from (Z)-8 (456 mg, 1.00 mmol), PhCHO (102 $\mu \mathrm{L}, 1.00$ mmol, 1.0 equiv) and $(R, R)-\mathbf{2 0 a}\left(37 \mathrm{mg}, 0.10 \mathrm{mmol}, 0.1\right.$ equiv) in $1 \mathrm{~mL}$ of $\mathrm{CH}_{2} \mathrm{Cl}_{2}$ was obtained $317 \mathrm{mg}(0.84 \mathrm{mmol}, 84 \%)$ of $\mathbf{1 2 a}$ as a clear, viscous oil after column chromatography (pentane/ether, 5/1, $\mathrm{SiO}_{2}$ ).

Data for (syn,anti)-12a:

${ }^{1} \underline{\mathrm{H} \mathrm{NMR}}: \quad\left(500 \mathrm{MHz}, \mathrm{CDCl}_{3}\right)$

$7.30(\mathrm{~d}, J=7.6,2 \mathrm{H}, \mathrm{HC}(9)) ; 7.17$ (t, $J=7.3,2 \mathrm{H}, \mathrm{HC}(8)) ; 7.08(\mathrm{t}, J=7.2,1 \mathrm{H}$, $\mathrm{HC}(10)) ; 5.04$ (t, $J=3.2,1 \mathrm{H}, \mathrm{HC}(1)) ; 4.44$ (sext, $J=6.1,1 \mathrm{H}, \mathrm{HC}(5)$ ); 2.74 (d, $J$ $=2.6,1 \mathrm{H}, \mathrm{HO}) ; 2.55(\mathrm{qd}, J=7.3,3.9,1 \mathrm{H}, \mathrm{HC}(2)) ; 2.46-2.21(\mathrm{ABX}, 2 \mathrm{H}$, $\left.\mathrm{H}_{2} \mathrm{C}(4)\right) ; 1.11\left(\mathrm{~d}, J=6.1,3 \mathrm{H}, \mathrm{H}_{3} \mathrm{C}(6)\right) ; 1.10$ - 1.02 (m, $\left.21 \mathrm{H}, \mathrm{H}_{3} \mathrm{C}(13), \mathrm{HC}(12)\right)$; $0.94\left(\mathrm{~d}, J=7.0,3 \mathrm{H}, \mathrm{H}_{3} \mathrm{C}(11)\right)$

${ }^{13}$ C NMR: $\quad\left(126 \mathrm{MHz}, \mathrm{CDCl}_{3}\right)$

214.83 (C(3)); 141.89 (C(7)); 128.50 (C(8)); 127.55 (C(10)); 126.12 (C(9)); 72.83 $(\mathrm{C}(5)) ; 65.40(\mathrm{C}(1)) ; 53.56(\mathrm{C}(2)) ; 52.21$ (C(4)); 24.29 (C(6)); 18.27 (C(13)); $12.60(\mathrm{C}(12)) ; 9.80(\mathrm{C}(11))$ 
IR: (neat)

3467 (br, m); 3064 (w); 3032 (w); 2962 (s); 2943 (s); 2893 (s); 2868 (s); 1705 (s); 1462 (s); 1375 (s); 1248 (m); 1213 (m); 1198 (m); 1128(s); 1097 (s); 1070 (m); $1012(\mathrm{~s})$

MS: $\quad(\mathrm{FAB})$

$379.3\left(\mathrm{M}^{+}, 13\right) ; 273.2$ (11); 230.2 (18); 229.2 (100); 202.2 (10); 201.2 (49); 185.1 (15); 175.1 (10); 159.1 (12); 157.1 (51); 154.1 (11), 137.0 (11); 136.0 (13); 131.1 (15); 115.1 (24); 107.0 (32); 105.0 (11), 103.0 (11)

Opt. Rot.: $\quad[\alpha]_{D}^{24}-25.9(\mathrm{c}=0.24, \mathrm{EtOH})$

TLC: $\quad R_{f} 0.17$ (pentane/ether, 5/1) [silica gel, $p$-anisaldehyde]

SFC: $\quad t_{\mathrm{R}} 3.645 \mathrm{~min}(\mathrm{OD}, 150 \mathrm{bar}, 3 \mathrm{~mL} / \mathrm{min}, 5 \% \mathrm{MeOH})$

Analysis: $\quad \mathrm{C}_{22} \mathrm{H}_{38} \mathrm{O}_{3} \mathrm{Si}$ (378.62)

Calcd: $\quad$ C, 69.79; H, 10.12\%

Found: $\quad \mathrm{C}, 69.63 ; \quad \mathrm{H}, 10.43 \%$

\section{(1S,2S,5S)-1-Triisopropylsilyloxy-2-methyl-5-hydroxy-5-phenyl-3-hexanone (syn, syn-12a)}<smiles></smiles>

$(Z)-8$
$\mathrm{PhCHO}$

a

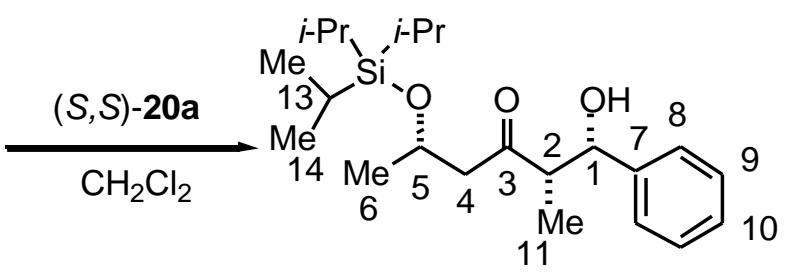

(syn,syn)-12a

Following General Procedure III from (Z)-8 (450 mg, $1.00 \mathrm{mmol})$, PhCHO (102 $\mu \mathrm{L}, 1.00$ mmol, 1.0 equiv) and $(S, S)$-20a (37 mg, $0.10 \mathrm{mmol}, 0.1$ equiv) in $1 \mathrm{~mL}$ of $\mathrm{CH}_{2} \mathrm{Cl}_{2}$ was obtained $327 \mathrm{mg}(0.86 \mathrm{mmol}, 86 \%)$ of $\mathbf{1 2 a}$ as a clear, colorless, viscous oil after column chromatography (pentane/ether, 5/1, $\mathrm{SiO}_{2}$ ). 
Data for $(s y n, s y n)-\mathbf{1 2 a}$ :

1슬 $: \quad\left(500 \mathrm{MHz}, \mathrm{CDCl}_{3}\right)$

$7.35-7.31$ (m, 4 H, HC(9), HC(8)); 7.27 (m, 1 H, HC(10)); 5.09 (t, J=3.4, 1 H, $\mathrm{HC}(1)) ; 4.40$ (sext, $J=5.9,1 \mathrm{H}, \mathrm{HC}(5)) ; 3.05$ (d, $J=2.7,1 \mathrm{H}, \mathrm{HO}) ; 2.85$ (qd, $J=$ 7.3, 3.9, $1 \mathrm{H}, \mathrm{HC}(2))$; 2.87-2.48 (ABX, $\left.2 \mathrm{H}, \mathrm{H}_{2} \mathrm{C}(4)\right) ; 1.15(\mathrm{~d}, J=6.1,3 \mathrm{H}$, $\left.\mathrm{H}_{3} \mathrm{C}(6)\right) ; 1.08-1.00\left(\mathrm{~m}, 24 \mathrm{H}, \mathrm{H}_{3} \mathrm{C}(13), \mathrm{HC}(12), \mathrm{H}_{3} \mathrm{C}(11)\right)$

${ }^{13}$ C NMR: $\quad\left(126 \mathrm{MHz}, \mathrm{CDCl}_{3}\right)$

214.48 (C(3)); $141.94(\mathrm{C}(7)) ; 128.51(\mathrm{C}(8)) ; 127.60$ (C(10)); $126.17(\mathrm{C}(9)) ; 73.11$ $(\mathrm{C}(5)) ; 65.13(\mathrm{C}(1)) ; 53.41(\mathrm{C}(2)) ; 52.27(\mathrm{C}(4)) ; 24.26(\mathrm{C}(6)) ; 18.30(\mathrm{C}(13))$; $12.59(\mathrm{C}(12)) ; 10.09(\mathrm{C}(11))$

IR: (neat)

3461 (br, m); 2963 (s); 2943 (s); 2893 (s); 2867 (s); 1707 (s); 1461 (m); 1375 (m); 1130 (s); 1097 (m); 1013 (s)

MS: (FI)

$379.2\left(\mathrm{M}^{+}, 4\right) ; 337.1$ (8); 336.1 (30); 335.1 (100); 272.2 (6); 229.2 (13); 157.1 (4); 156.8 (4), 156.7 (5); $156.6(6)$

Opt. Rot.: $\quad[\alpha]_{\mathrm{D}}^{24}+29.1(\mathrm{c}=0.59$, EtOH $)$

TLC: $\quad R_{f} 0.17$ (pentane/ether, 5/1) [silica gel, $p$-anisaldehyde]

SFC: $\quad t_{\mathrm{R}} 4.452 \mathrm{~min}(\mathrm{OD}, 150 \mathrm{bar}, 3 \mathrm{~mL} / \mathrm{min}, 5 \% \mathrm{MeOH})$

Analysis: $\quad \mathrm{C}_{22} \mathrm{H}_{38} \mathrm{O}_{3} \mathrm{Si}(378.62)$
Calcd:
C, 69.79;
H, $10.12 \%$

Found:

C, 69.54;

H, $10.32 \%$ 


\section{(1R,2R,5S)-5-Triisopropylsilyloxy-2-methyl-1-hydroxy-1-(1-naphthyl)-3-hexanone} (syn,anti-12b)

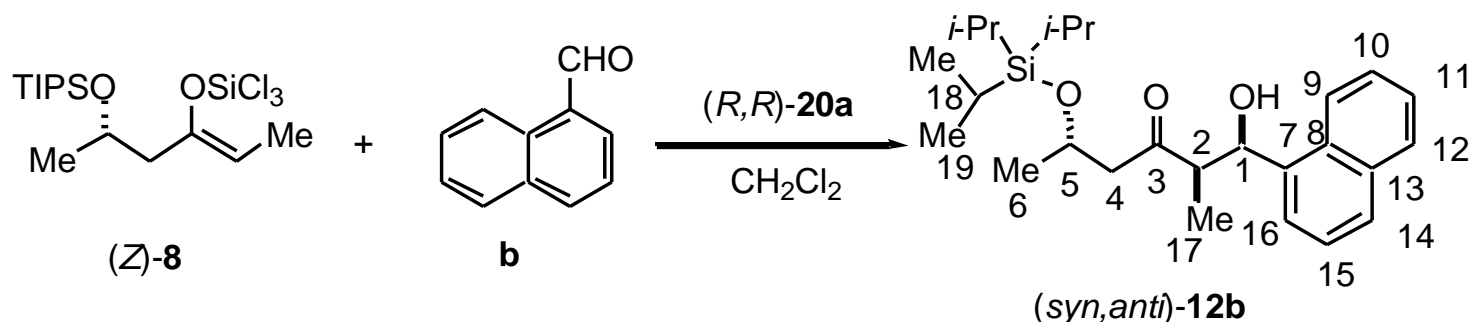

Following General Procedure III from (Z)-8 (451 mg, $1.00 \mathrm{mmol})$, 1-naphthaldehyde $(136 \mu \mathrm{L}, 1.00 \mathrm{mmol}, 1.0$ equiv) and $(R, R)-\mathbf{2 0 a}(37 \mathrm{mg}, 0.10 \mathrm{mmol}, 0.1$ equiv) in $1 \mathrm{~mL}$ of $\mathrm{CH}_{2} \mathrm{Cl}_{2}$ for $8 \mathrm{~h}$, was obtained $356 \mathrm{mg}(0.83 \mathrm{mmol}, 83 \%)$ of $\mathbf{1 2 b}$ as a clear, colorless, viscous oil after column chromatography (pentane/ether, $5 / 1, \mathrm{SiO}_{2}$ ).

Data for (syn,anti)-12b:

1슬 $\quad\left(500 \mathrm{MHz}, \mathrm{CDCl}_{3}\right)$

7.89 (d, $J=6.2,1 \mathrm{H}, \mathrm{HC}(9)) ; 7.87$ (d, $J=5.9,1 \mathrm{H}, \mathrm{HC}(12)) ; 7.78$ (d, $J=8.0,1 \mathrm{H}$, $\mathrm{HC}(14)) ; 7.72$ (d, $J=7.3,1 \mathrm{H}, \mathrm{HC}(16)) ; 7.52-7.47$ (m, $3 \mathrm{H}, \mathrm{HC}(11), \mathrm{HC}(10)$, $\mathrm{HC}(15)) ; 5.96$ (t, $J=2.4,1 \mathrm{H}, \mathrm{HC}(1)) ; 4.48$ (sext, $J=6.4,1 \mathrm{H}, \mathrm{HC}(5)$ ); 3.26 (d, $J$ $=2.4,1 \mathrm{H}, \mathrm{HO}) ; 3.03(\mathrm{qd}, J=7.1,4.4,1 \mathrm{H}, \mathrm{HC}(2)) ; 2.81-2.61(\mathrm{ABX}, 2 \mathrm{H}$, $\left.\mathrm{H}_{2} \mathrm{C}(4)\right) ; 1.22\left(\mathrm{~d}, J=5.8, \mathrm{H}_{3} \mathrm{C}(6)\right) ; 1.10-1.04\left(\mathrm{~m}, 24 \mathrm{H}, \mathrm{H}_{3} \mathrm{C}(17), \mathrm{HC}(18)\right.$, $\left.\mathrm{H}_{3} \mathrm{C}(19)\right)$

${ }^{13}$ C NMR: $\quad\left(126 \mathrm{MHz}, \mathrm{CDCl}_{3}\right)$

214.42 (C(3)); $136.66(\mathrm{C}(7)) ; 133.68$ (C(13)); 129.62 (C(8)); 129.11 (C(12)); 127.83 (C(14)); 126.04 (C(10)); 125.35 (C(11)); 125.34 (C(15)); 124.27 (C(16)); 122.37 (C(9)); 69.07 (C(1)); 64.85 (C(5); 51.88 (C(4)); 51.40 (C(2)); 24.08 (C(6)); 18.03 (C(19)); 12.33 (C(18)); 9.62 (C(17))

IR: $\quad\left(\mathrm{CH}_{2} \mathrm{Cl}_{2}\right)$

3468 (br, m); 2962 (s); 2944 (s); 2892 (s); 2867 (s); 1704 (s); 1462 (s); 1374 (s); 1133 (s); 1102 (s); 1056 (m); 1029 (s); 1013 (s) 
MS: $\quad(\mathrm{FI})$

$429.2\left(\mathrm{M}^{+}, 4\right) ; 411.3(7) ; 410.3$ (13); 387.2 (7); 387.0 (6); 386.1 (28); 385.1 (100);

384.2 (4); 299.2 (4); 282.2 (4); 274.2 (4); 273.2 (15); 272.2 (57); 256.2 (4); 254.2 (4); 231.2 (4); 230.1 (8); 229.1 (58); 192.6 (5); 157.1 (8); 156.0 (44); 136.4 (5); $136.0(5)$

Opt. Rot.: $\quad[\alpha]_{\mathrm{D}}^{24}-4.73(\mathrm{c}=0.61, \mathrm{EtOH})$

TLC: $\quad R_{f} 0.09$ (pentane/ether, 5/1) [silica gel, short uv]

SFC: $\quad t_{\mathrm{R}} 5.940 \mathrm{~min}(\mathrm{OD}, 150 \mathrm{bar}, 3 \mathrm{~mL} / \mathrm{min}, 15 \% \mathrm{MeOH})$

Analysis: $\quad \mathrm{C}_{26} \mathrm{H}_{40} \mathrm{O}_{3} \mathrm{Si}(428.68)$
Calcd:
C, 72.85;
$\mathrm{H}, 9.41 \%$
Found:
C, 72.68;
$\mathrm{H}, 9.49 \%$

(1S,2S,5S)-5-Triisopropylsilyloxy-2-methyl-1-hydroxy-1-(1-naphthyl)-3-hexanone (syn,syn12b)<smiles>C/C=C(/CC(C)OC(C)(F)F)O[Na]</smiles>

$(Z)-8$<smiles>O=Cc1cccc2ccccc12</smiles>

b

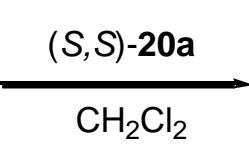

$\mathrm{CH}_{2} \mathrm{Cl}_{2}$

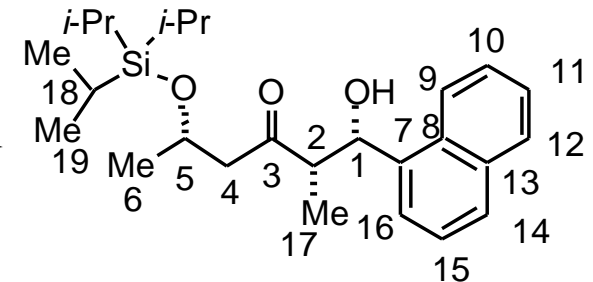

(syn,syn)-12b

Following General Procedure III from $(Z)-8$ (451 mg, $1.00 \mathrm{mmol})$, 1-naphthaldehyde $\left(136 \mu \mathrm{L}, 1.00 \mathrm{mmol}, 1.0\right.$ equiv) and $(S, S)-\mathbf{2 0 a}\left(37 \mathrm{mg}, 0.10 \mathrm{mmol}, 0.1\right.$ equiv) in $1 \mathrm{~mL}$ of $\mathrm{CH}_{2} \mathrm{Cl}_{2}$ for $8 \mathrm{~h}$, was obtained $347 \mathrm{mg}(0.75 \mathrm{mmol}, 75 \%)$ of 12b as a clear, colorless, viscous oil after column chromatography (pentane/ether, $5 / 1, \mathrm{SiO}_{2}$ ). 
Data for $(s y n, s y n)-\mathbf{1 2 b}$ :

1슬 $: \quad\left(500 \mathrm{MHz}, \mathrm{CDCl}_{3}\right)$

7.88 (d, $J=7.4,1 \mathrm{H}, \mathrm{HC}(9)) ; 7.87$ (d, $J=4.1,1 \mathrm{H}, \mathrm{HC}(12)) ; 7.79$ (d, $J=8.0,1 \mathrm{H}$, $\mathrm{HC}(14)$ ); 7.72 (d, $J=7.1,1 \mathrm{H}, \mathrm{HC}(16))$; 7.52-7.48 (m, $3 \mathrm{H}, \mathrm{HC}(11), \mathrm{HC}(10)$, $\mathrm{HC}(15)$ ); 5.94 (dd, $J=3.4,2.5,1 \mathrm{H}, \mathrm{HC}(1)) ; 4.46$ (sext, $J=6.1,1 \mathrm{H}, \mathrm{HC}(5)) ; 3.24$ (d, $J=2.4,1 \mathrm{H}, \mathrm{HO}) ; 3.04$ (qd, $J=7.3,4.4,1 \mathrm{H}, \mathrm{HC}(2)) ; 2.87$ - 2.57 (ABX, $2 \mathrm{H}$, $\left.\mathrm{H}_{2} \mathrm{C}(4)\right) ; 1.21\left(\mathrm{~d}, J=6.1, \mathrm{H}_{3} \mathrm{C}(6)\right) ; 1.09-1.02$ (m, $24 \mathrm{H}, \mathrm{H}_{3} \mathrm{C}(17), \mathrm{HC}(18)$, $\left.\mathrm{H}_{3} \mathrm{C}(19)\right)$

${ }^{13}$ C NMR: $\quad\left(126 \mathrm{MHz}, \mathrm{CDCl}_{3}\right)$

$214.31(\mathrm{C}(3))$; $136.63(\mathrm{C}(7)) ; 133.68(\mathrm{C}(13)) ; 129.61(\mathrm{C}(8)) ; 129.12(\mathrm{C}(12))$; 127.86 (C(14)); 126.09 (C(10)); 125.37 (C(11)); 125.34 (C(15)); 124.12 (C(16)); 122.31 (C(9)); 69.17 (C(1)); 64.90 (C(5)); 51.82 (C(4)); 51.38 (C(2)); 24.11 (C(6)); 17.99 (C(19)); 12.33 (C(18)); 9.59 (C(17))

IR: (neat)

3470 (br, m); 2963 (s); 2943 (s); 2892 (m); 2867 (s); 1704 (m); 1462 (m); 1374 (m); $1130(\mathrm{~m}) ; 1104(\mathrm{~m}) ; 1013$ (s)

MS: (FI)

$429.2\left(\mathrm{M}^{+}, 16\right) ; 428.1$ (10); 386.1 (28); 385.1 (95); 321.1 (17); 303.1 (11); 273.1 (21); 272.1 (58); 230.1 (17); 229.1 (97); 157.0 (20); 156.0 (100)

Opt. Rot.: $\quad[\alpha]_{\mathrm{D}}^{24}+14.2(\mathrm{c}=0.63$, EtOH $)$

TLC: $\quad R_{f} 0.09$ (pentane/ether, 5/1) [silica gel, short uv]

SFC: $\quad t_{\mathrm{R}} 3.562 \mathrm{~min}(\mathrm{OD}, 150 \mathrm{bar}, 3 \mathrm{~mL} / \mathrm{min}, 15 \% \mathrm{MeOH})$

Analysis: $\quad \mathrm{C}_{26} \mathrm{H}_{40} \mathrm{O}_{3} \mathrm{Si}(428.68)$

Calcd: $\quad$ C, $72.85 ; \quad$ H, $9.41 \%$

Found: $\quad$ C, $73.02 ; \quad H, 9.78 \%$ 
(2S,5R,6R)-2-Triisopropylsilyloxy-5-methyl-6-hydroxy-8-phenyl-7-octen-4-one (syn,anti-

12c)

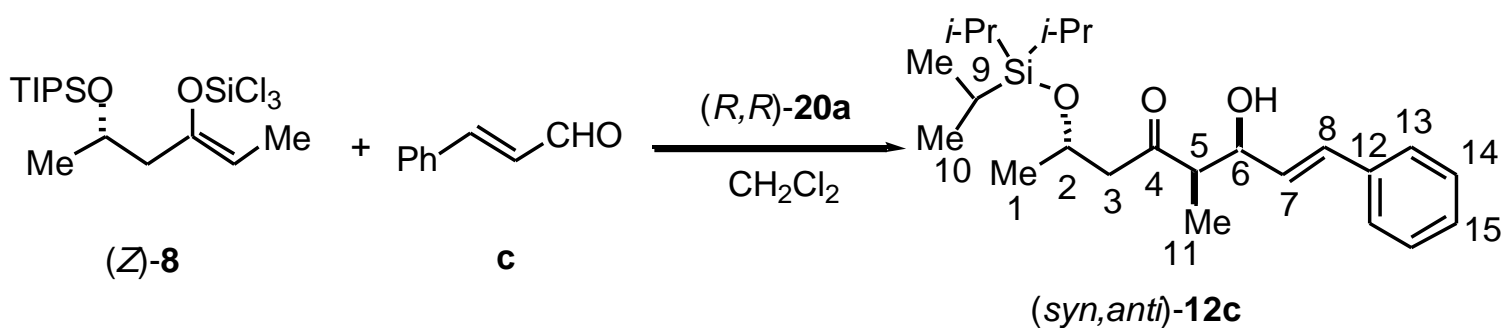

Following General Procedure III from $(Z)-8$ (451 mg, $1.00 \mathrm{mmol})$, cinnamaldehyde (126 $\mu \mathrm{L}, 1.00 \mathrm{mmol}, 1.0$ equiv) and (R,R)-20a (37 mg, $0.10 \mathrm{mmol}, 0.1$ equiv) in $1 \mathrm{~mL}$ of $\mathrm{CH}_{2} \mathrm{Cl}_{2}$ for $12 \mathrm{~h}$, was obtained $322 \mathrm{mg}(0.80 \mathrm{mmol}, 80 \%)$ of 12c as a clear, colorless, viscous oil after column chromatography (pentane/ether, $2 / 1, \mathrm{SiO}_{2}$ ).

Data for (syn,anti)-12c:

1슬 $\quad$ (500 MHz, $\left.\mathrm{CDCl}_{3}\right)$

$7.37(\mathrm{~d}, J=7.3,2 \mathrm{H}, \mathrm{HC}(13)) ; 7.31(\mathrm{t}, J=7.6,2 \mathrm{H}, \mathrm{HC}(14)) ; 7.24(\mathrm{t}, J=7.1,1$ $\mathrm{H}, \mathrm{HC}(15)) ; 6.64$ (d, $J=15.9,1 \mathrm{H}, \mathrm{HC}(8)) ; 6.14$ (dd, $J=15.8,5.9,1 \mathrm{H}, \mathrm{HC}(7))$; 4.67 (m, $1 \mathrm{H}, \mathrm{HC}(6)) ; 4.45$ (sext, $J=6.1,1 \mathrm{H}, \mathrm{HC}(2)) ; 2.88$ (d, J = 3.2, $1 \mathrm{H}, \mathrm{HO}$ ); 2.82-2.59 (ABX, $\left.2 \mathrm{H}, \mathrm{H}_{2} \mathrm{C}(3)\right) ; 2.76$ (qd, $\left.J=7.3,3.4,1 \mathrm{H}, \mathrm{HC}(5)\right) ; 1.22$ (d, $J$ $\left.=6.1,3 \mathrm{H}, \mathrm{H}_{3} \mathrm{C}(1)\right) ; 1.17\left(\mathrm{~d}, J=7.1,3 \mathrm{H}, \mathrm{H}_{3} \mathrm{C}(11)\right) ; 1.05$ (m, $21 \mathrm{H}, \mathrm{HC}(9)$, $\left.\mathrm{H}_{3} \mathrm{C}(10)\right)$

${ }^{13}$ C NMR: $\quad\left(126 \mathrm{MHz}, \mathrm{CDCl}_{3}\right)$

$219.29(\mathrm{C}(4)) ; 136.63(\mathrm{C}(12)) ; 131.06(\mathrm{C}(7)) ; 128.90(\mathrm{C}(8)) ; 128.57(\mathrm{C}(14))$; 127.66 (C(15)); $126.46(\mathrm{C}(13)) ; 71.81$ (C(6)); 65.33 (C(2)); $52.00(\mathrm{C}(3)) ; 51.53$ $(\mathrm{C}(5)) ; 24.18(\mathrm{C}(1)) ; 18.07$ (C(10)); 12.39 (C(9)); 10.02 (C(11))

IR: (neat) 3442 (br, m); 2963 (s); 2943 (s); 2892 (s); 2867 (s); 1705 (s); 1462 (m); 1374 (m); $1126(\mathrm{~s}) ; 1013$ (s)

MS: $\quad(\mathrm{FI})$

$404.1\left(\mathrm{M}^{+}, 9\right) ; 361.1$ (7); 321.1 (2); 272.1 (13); 229.0 (100); 132.0 (86) 
Opt. Rot.: $\quad[\alpha]_{\mathrm{D}}^{24}-20.5(\mathrm{c}=0.33, \mathrm{EtOH})$

TLC: $\quad R_{f} 0.21$ (pentane/ether, 2/1) [silica gel, short uv]

SFC: $\quad t_{\mathrm{R}} 2.348 \mathrm{~min}(\mathrm{OD}, 150 \mathrm{bar}, 3 \mathrm{~mL} / \mathrm{min}, 15 \% \mathrm{MeOH})$

Analysis: $\quad \mathrm{C}_{24} \mathrm{H}_{40} \mathrm{O}_{3} \mathrm{Si}(404.66)$
Calcd:
C, 71.23;
H, $9.96 \%$
Found:
C, 71.20;
$\mathrm{H}, 10.21 \%$

(2S,5S,6S)-2-Triisopropylsilyloxy-5-methyl-6-hydroxy-8-phenyl-7-octen-4-one (syn,syn-12c)

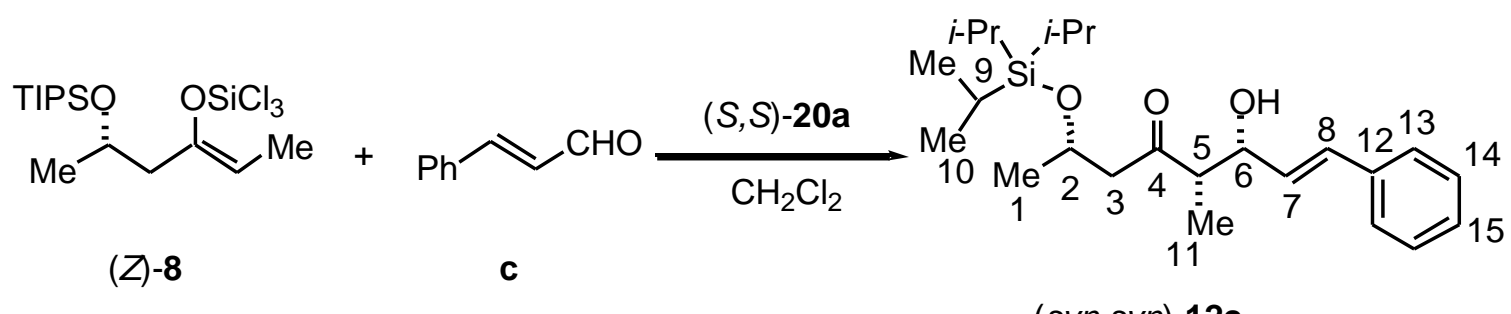

(syn,syn)-12c

Following General Procedure III from $(Z)-8$ (451 mg, $1.00 \mathrm{mmol})$, cinnamaldehyde (126 $\mu \mathrm{L}, 1.00 \mathrm{mmol}, 1.0$ equiv) and $(S, S)-1 \mathbf{a}\left(37 \mathrm{mg}, 0.10 \mathrm{mmol}, 0.1\right.$ equiv) in $1 \mathrm{~mL}$ of $\mathrm{CH}_{2} \mathrm{Cl}_{2}$ for 12 h, was obtained $304 \mathrm{mg}(0.75 \mathrm{mmol}, 75 \%)$ of 12c as a clear, colorless, viscous oil after column chromatography (pentane/ether 2/1).

Data for $(s y n, s y n)-\mathbf{1 2 c}$ :

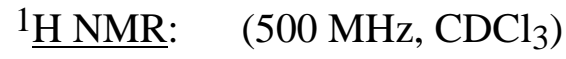

$7.37(\mathrm{~d}, J=7.1,2 \mathrm{H}, \mathrm{HC}(13)) ; 7.31(\mathrm{t}, J=7.8,2 \mathrm{H}, \mathrm{HC}(14)) ; 7.24(\mathrm{t}, J=7.4,1$ $\mathrm{H}, \mathrm{HC}(15)) ; 6.64(\mathrm{dd}, J=15.9,1.2,1 \mathrm{H}, \mathrm{HC}(8)) ; 6.15$ (dd, $J=15.8,5.8,1 \mathrm{H}$, $\mathrm{HC}(7)) ; 4.66$ (m, $1 \mathrm{H}, \mathrm{HC}(6)) ; 4.45$ (sext, $J=5.6,1 \mathrm{H}, \mathrm{HC}(2)) ; 2.85$ (d, J = 3.2, 1 H, HO); 2.78 (m, $1 \mathrm{H}, \mathrm{HC}(5))$; 2.80-2.62 (ABX, $\left.2 \mathrm{H}, \mathrm{H}_{2} \mathrm{C}(3)\right)$; 1.22 (d, J =6.1, 3 $\left.\mathrm{H}, \mathrm{H}_{3} \mathrm{C}(1)\right) ; 1.18$ (d, $\left.J=7.3,3 \mathrm{H}, \mathrm{H}_{3} \mathrm{C}(11)\right) ; 1.08-1.03$ (m, $21 \mathrm{H}, \mathrm{HC}(9)$, $\left.\mathrm{H}_{3} \mathrm{C}(10)\right)$ 
${ }^{13} \underline{\mathrm{C} \mathrm{NMR}}: \quad\left(126 \mathrm{MHz}, \mathrm{CDCl}_{3}\right)$

213.97 (C(4)); $136.61(\mathrm{C}(12)) ; 131.13(\mathrm{C}(7)) ; 128.96(\mathrm{C}(8)) ; 128.57$ (C(14)); 127.67 (C(15)); $126.46(\mathrm{C}(13)) ; 72.00(\mathrm{C}(6))$; $65.12(\mathrm{C}(2)) ; 51.97(\mathrm{C}(3)) ; 51.44$ $(\mathrm{C}(5)) ; 24.06(\mathrm{C}(1)) ; 18.06(\mathrm{C}(10)) ; 12.38(\mathrm{C}(9)) ; 10.16(\mathrm{C}(11))$

IR: (neat) 3436 (br, m); 2963 (s); 2943 (s); 2892 (m); 2867 (s); 1707 (m); 1462 (m); 1374 $(\mathrm{m}) ; 1130(\mathrm{~m}) ; 1013(\mathrm{~s})$

MS: $\quad(\mathrm{FI})$

$404.1\left(\mathrm{M}^{+}, 18\right) ; 385.1$ (8); 361.1 (7); 321.0 (9); 273.1 (10); 272.1 (21); 245.0 (9); 230.1 (19); 229.1 (100); 156.0 (8); 133.0 (12); 132.0 (74)

Opt. Rot.: $\quad[\alpha]_{\mathrm{D}}^{24}+27.7(\mathrm{c}=0.50, \mathrm{EtOH})$

TLC: $\quad R_{f} 0.20$ (pentane/ether, 2/1) [silica gel, short uv]

SFC: $\quad t_{\mathrm{R}} 3.231 \mathrm{~min}(\mathrm{OD}, 150 \mathrm{bar}, 3 \mathrm{~mL} / \mathrm{min}, 15 \% \mathrm{MeOH})$

Analysis: $\quad \mathrm{C}_{24} \mathrm{H}_{40} \mathrm{O}_{3} \mathrm{Si}$ (404.66)

Calcd: $\quad$ C, $71.23 ; \quad$ H, $9.96 \%$

Found: $\quad$ C, $71.29 ; \quad \mathrm{H}, 10.31 \%$

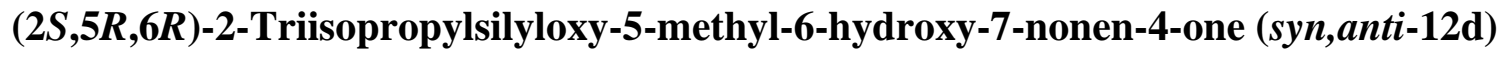

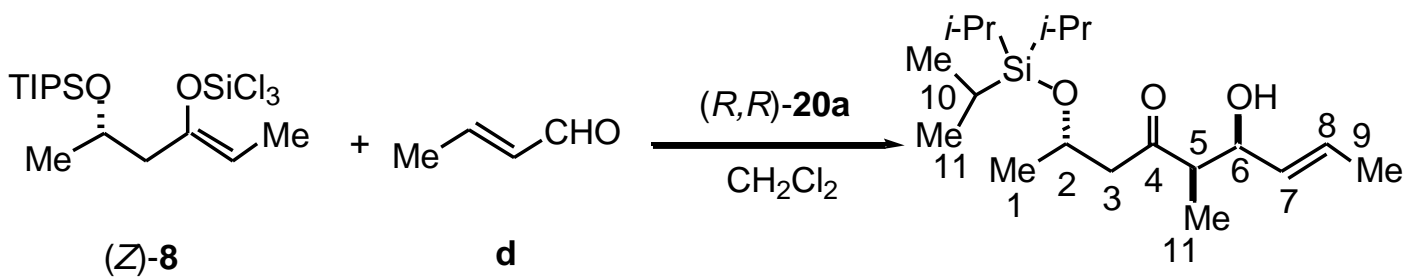

(syn, anti)-12d

Following General Procedure III from $(Z)-8$ (451 mg, $1.00 \mathrm{mmol})$, crotonaldehyde (83 $\mu \mathrm{L}, 1.00 \mathrm{mmol}, 1.0$ equiv) and $(R, R)-\mathbf{2 0 a}\left(37 \mathrm{mg}, 0.10 \mathrm{mmol}, 0.1\right.$ equiv) in $1 \mathrm{~mL}$ of $\mathrm{CH}_{2} \mathrm{Cl}_{2}$ for $8 \mathrm{~h}$, was obtained $272 \mathrm{mg}(0.79 \mathrm{mmol}, 79 \%)$ of $\mathbf{1 2 d}$ as a clear, colorless, viscous oil after column chromatography (pentane/ether, $3 / 1, \mathrm{SiO}_{2}$ ). 


\section{Data for (syn,anti)-12d:}

1ㅂ NMR: $\quad\left(500 \mathrm{MHz}\right.$, benzene- $\left.d_{6}\right)$

$5.61(\mathrm{dqd}, J=15.2,6.4,1.2,1 \mathrm{H}, \mathrm{HC}(8)) ; 5.37$ (ddd, $J=15.4,6.1,1.5,1 \mathrm{H}$, $\mathrm{HC}(7)$ ); 4.51 (sext, $J=6.3,1 \mathrm{H}, \mathrm{HC}(2)) ; 4.31$ (m, $1 \mathrm{H}, \mathrm{HC}(6)) ; 2.68-2.34$ (ABX, $\left.2 \mathrm{H}, \mathrm{H}_{2} \mathrm{C}(3)\right) ; 2.36(\mathrm{~m}, 1 \mathrm{H}, \mathrm{HC}(5)) ; 2.10(\mathrm{~d}, J=3.5,1 \mathrm{H}, \mathrm{HO}) ; 1.53$ (d, $J=6.5$, $\left.3 \mathrm{H}, \mathrm{H}_{3} \mathrm{C}(9)\right) ; 1.17$ (d, $\left.J=6.1,3 \mathrm{H}, \mathrm{H}_{3} \mathrm{C}(1)\right) ; 1.15-1.03$ (m, $21 \mathrm{H}, \mathrm{H}_{3} \mathrm{C}(11)$, $\mathrm{HC}(10)) ; 1.01$ (d, $\left.J=7.3,3 \mathrm{H}, \mathrm{H}_{3} \mathrm{C}(12)\right)$

${ }^{13}$ C NMR: $\quad\left(126 \mathrm{MHz}, \mathrm{CDCl}_{3}\right)$

$214.08(\mathrm{C}(4)) ; 130.48(\mathrm{C}(7)) ; 127.87(\mathrm{C}(8) ; 72.16(\mathrm{C}(6)) ; 65.15(\mathrm{C}(2)) ; 52.07$ $(\mathrm{C}(3))$; $51.63(\mathrm{C}(5)) ; 24.09$ (C(1)); 18.02 (C(11)); 17.67 (C(9)); 12.32 (C(10)); $10.19(\mathrm{C}(12))$

IR: (neat)

3442 (br, m); 2963 (s); 2943 (s); 2893 (s); 2868 (s); 1710 (s); 1463 (m); 1375 (m); 1130 (s); $1102(\mathrm{~m}) ; 1013$ (s)

$\underline{\mathrm{MS}}: \quad(\mathrm{FI})$

$343.2\left(\mathrm{M}^{+}, 3\right) ; 301.2$ (7); 300.2 (22); 299.6 (4); 299.2 (100); 281.2 (4); 272.2 (8); 230.1 (6); 29.1 (36); 176.2 (3); 176.1 (4); 176.0 (4); 175.9 (5); 175.8 (6); 175.6 (6); 175.4 (5); $175.3(5) ; 175.1$ (4)

Opt. Rot:: $\quad[\alpha]_{\mathrm{D}}^{24}-15.9(\mathrm{c}=0.54, \mathrm{EtOHc}=0.54)$

TLC: $\quad R_{f} 0.16$ (pentane/ether, 3/1) [silica gel, $p$-anisaldehyde]

Analysis: $\quad \mathrm{C}_{19} \mathrm{H}_{38} \mathrm{O}_{3} \mathrm{Si}(342.59)$

Calcd: $\quad$ C, 66.61; H, $11.18 \%$

Found: $\quad$ C, $66.37 ; \quad H, 11.36 \%$ 


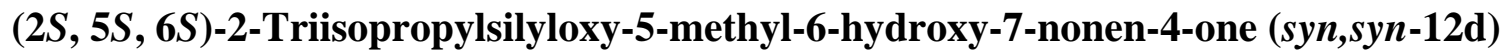

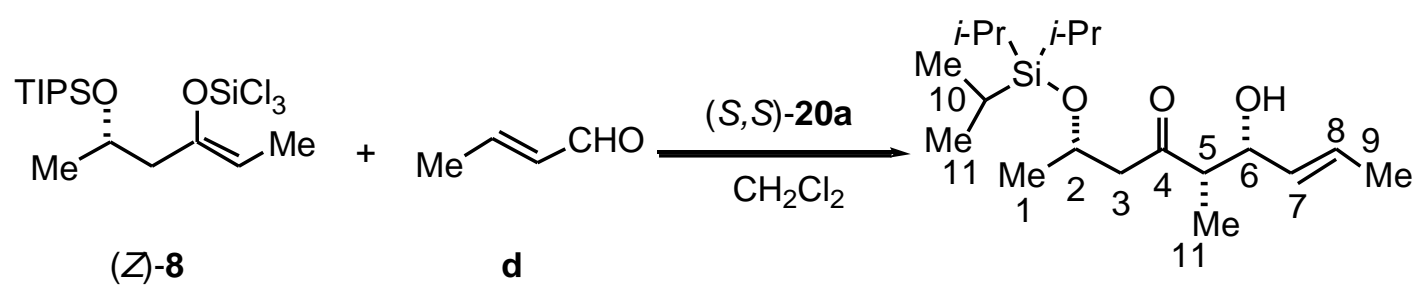

(syn,syn)-12d

Following General Procedure III from $(Z)-8(451 \mathrm{mg}, 1.00 \mathrm{mmol})$, crotonaldehyde (83 $\mu \mathrm{L}, 1.00 \mathrm{mmol}, 1.0$ equiv) and (S,S)-20a (37 mg, $0.10 \mathrm{mmol}, 0.1$ equiv) in $1 \mathrm{~mL}$ of $\mathrm{CH}_{2} \mathrm{Cl}_{2}$ for 8 h, was obtained $285 \mathrm{mg}(0.83 \mathrm{mmol}, 83 \%)$ of $\mathbf{1 2 d}$ as a clear, colorless, viscous oil after column chromatography (pentane/ether, $3 / 1, \mathrm{SiO}_{2}$ ).

Data for $(s y n, s y n)-\mathbf{1 2 d}$ :

${ }^{1}$ H NMR: $\quad\left(500 \mathrm{MHz}\right.$, benzene- $\left.d_{6}\right)$

$5.58(\mathrm{dqd}, J=15.4,6.4,1.2,1 \mathrm{H}, \mathrm{HC}(8)) ; 5.35(\mathrm{ddd}, J=15.4,6.1,1.7,1 \mathrm{H}$, $\mathrm{HC}(7)$ ); 4.51 (sext, $J=5.9,1 \mathrm{H}, \mathrm{HC}(2)) ; 4.25$ (m, $1 \mathrm{H}, \mathrm{HC}(6)$ ); 2.68 - 2.34 (ABX, $\left.2 \mathrm{H}, \mathrm{H}_{2} \mathrm{C}(3)\right) ; 2.38(\mathrm{~m}, 1 \mathrm{H}, \mathrm{HC}(5)) ; 2.00(\mathrm{~d}, J=3.4,1 \mathrm{H}, \mathrm{HO}) ; 1.51$ (dd, $J=6.4$, $\left.1.0,3 \mathrm{H}, \mathrm{H}_{3} \mathrm{C}(9)\right) ; 1.18$ (d, $\left.J=6.1,3 \mathrm{H}, \mathrm{H}_{3} \mathrm{C}(1)\right) ; 1.16$ - 1.00 (m, $21 \mathrm{H}, \mathrm{H}_{3} \mathrm{C}(11)$, $\mathrm{HC}(10)) ; 1.05$ (d, $\left.J=7.1,3 \mathrm{H}, \mathrm{H}_{3} \mathrm{C}(12)\right)$

${ }^{13} \mathrm{C} \mathrm{NMR}: \quad\left(126 \mathrm{MHz}, \mathrm{CDCl}_{3}\right)$

$213.71(\mathrm{C}(4)) ; 130.57(\mathrm{C}(7)) ; 127.97(\mathrm{C}(8) ; 72.41(\mathrm{C}(6)) ; 64.95(\mathrm{C}(2)) ; 52.15$ (C(3)); 51.56 (C(5)); 24.04 (C(1)); 17.97 (C(11)); 17.66 (C(9)); 12.30 (C(11)); $10.36(\mathrm{C}(12))$

IR: (neat)

3441 (br, m); 2963 (s); 2944 (m); 2893 (m); 2867 (s); 1710 (m); 1462 (m); 1375 (m); $1132(\mathrm{~m}) ; 1109(\mathrm{~m}) ; 1013(\mathrm{~m})$

$\underline{\mathrm{MS}}: \quad(\mathrm{FI})$

$343.2(2) ; 342.2\left(\mathrm{M}^{+}, 3\right) ; 301.2$ (7); 300.2 (22); 299.2 (100); 229.2 (37); 175.3 (5)

Opt. Rot.: $\quad[\alpha]_{\mathrm{D}}^{24}+30.4(\mathrm{c}=0.54$, EtOH $)$

TLC: $\quad R_{f} 0.16$ (pentane/ether, 3/1) [silica gel, $p$-anisaldehyde] 
Analysis: $\quad \mathrm{C}_{19} \mathrm{H}_{38} \mathrm{O}_{3} \mathrm{Si}(342.59)$

$\begin{array}{lll}\text { Calcd: } & \text { C }, 66.61 ; & \text { H }, 11.18 \% \\ \text { Found: } & \text { C, 66.33; } & \text { H, } 11.33 \%\end{array}$

(2S,5R,6R)-2-Triisopropylsilyloxy-11,12-dimethyl-6-hydroxy-8-phenyl-7-octen-4-one (syn,anti-12e)

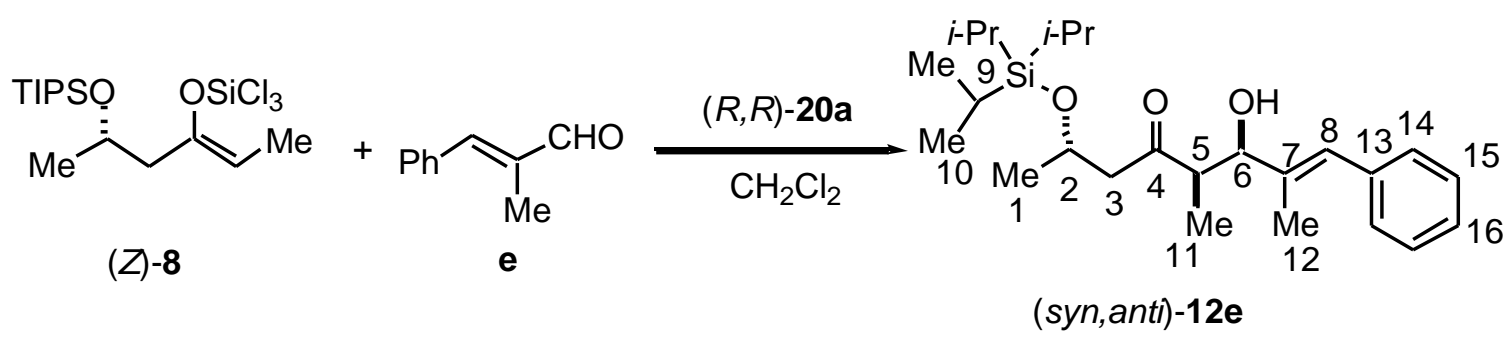

Following General Procedure III from (Z)-8 (436 mg, $1.00 \mathrm{mmol}), \quad \alpha-$ methylcinnamaldehyde $(140 \mu \mathrm{L}, 1.00 \mathrm{mmol}, 1.0$ equiv) and $(R, R)-\mathbf{2 0 a}(37 \mathrm{mg}, 0.10 \mathrm{mmol}, 0.1$ equiv) in $1 \mathrm{~mL}$ of $\mathrm{CH}_{2} \mathrm{Cl}_{2}$ for $10 \mathrm{~h}$, was obtained $332 \mathrm{mg}(0.79 \mathrm{mmol}, 79 \%)$ of $\mathbf{1 2 e}$ as a clear viscous oil after column chromatography (pentane/ether, 3/1, $\mathrm{SiO}_{2}$ ).

Data for (syn,anti)-12e:

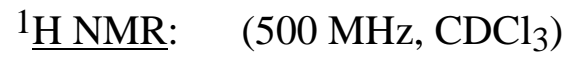

$7.33(\mathrm{t}, J=7.8,2 \mathrm{H}, \mathrm{HC}(15)) ; 7.27(\mathrm{~d}, J=7.3,2 \mathrm{H}, \mathrm{HC}(14)) ; 7.21(\mathrm{t}, J=7.0,1$ $\mathrm{H}, \mathrm{HC}(16)$ ); 6.63 (br s, $1 \mathrm{H}, \mathrm{HC}(8)$ ); 4.53 (m, $1 \mathrm{H}, \mathrm{HC}(6)$ ); 4.47 (sext, $J=6.3,1$ $\mathrm{H}, \mathrm{HC}(2)) ; 2.99$ (d, $J=2.7,1 \mathrm{H}, \mathrm{HO}) ; 2.84-2.62\left(\mathrm{ABX}, 2 \mathrm{H}, \mathrm{H}_{2} \mathrm{C}(3)\right) ; 2.82$ (m, 1 $\mathrm{H}, \mathrm{HC}(5)) ; 1.81\left(\mathrm{~d}, J=1.0,3 \mathrm{H}, \mathrm{H}_{3} \mathrm{C}(12)\right) ; 1.22$ (d, $\left.J=5.9,3 \mathrm{H}, \mathrm{H}_{3} \mathrm{C}(1)\right) ; 1.13$ (d, $\left.J=7.3,3 \mathrm{H}, \mathrm{H}_{3} \mathrm{C}(11)\right) ; 1.07-1.00\left(\mathrm{~m}, 21 \mathrm{H}, \mathrm{HC}(9), \mathrm{H}_{3} \mathrm{C}(10)\right)$

${ }^{13} \mathrm{C} \mathrm{NMR}: \quad\left(126 \mathrm{MHz}, \mathrm{CDCl}_{3}\right)$

214.31 (C(4)); 137.64 (C(13)); 136.34 (C(7)); 128.99 (C(15)); 128.07 (C(14)); 126.35 (C(16)); 125.86 (C(8)); 74.77 (C(6)); 65.28 (C(2)); 51.69 (C(3)); 49.21 (C(5)); 24.11 (C(1)); 18.02 (C(10)); 15.26 (C(12)); 12.36 (C(9)); 9.27 (C(11)) 
IR: $\quad\left(\mathrm{CHCl}_{3}\right)$

3487 (br, w); 2962 (m); 2945 (m); 2868 (m); 1699 (w); 1464 (w); 1377 (w); 1101 (w)

MS: $\quad(\mathrm{FI})$

$418.2\left(\mathrm{M}^{+}, 100\right) ; 375.2(25) ; 336.7$ (8); $272.2(22) ; 229.1$ (27); $187.6(4) ; 146.1$ (29); $115.1(2)$

Opt. Rot.: $\quad[\alpha]_{\mathrm{D}}^{24}-2.13(\mathrm{c}=0.63, \mathrm{EtOH})$

TLC: $\quad R_{f} 0.22$ (pentane/ether, 3/1) [silica gel, short uv]

SFC: $\quad t_{\mathrm{R}} 2.03 \mathrm{~min}(\mathrm{OD}, 150 \mathrm{bar}, 3 \mathrm{~mL} / \mathrm{min}, 15 \% \mathrm{MeOH})$

Analysis: $\quad \mathrm{C}_{25} \mathrm{H}_{42} \mathrm{O}_{3} \mathrm{Si}(418.68)$

Calcd: $\quad$ C, $71.72 ; \quad H, 10.11 \%$

Found: $\quad$ C, $71.34 ; \quad H, 10.18 \%$

\section{(2S,5S,6S)-2-Triisopropylsilyloxy-11,12-dimethyl-6-hydroxy-8-phenyl-7-octen-4-one} (syn,syn-12e)

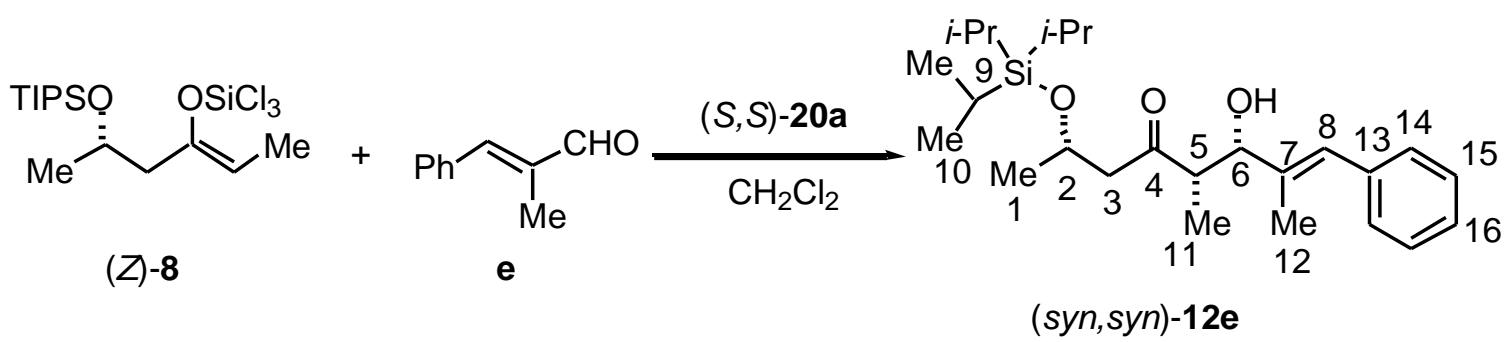

Following General Procedure III from (Z)-8 (436 mg, $1.00 \mathrm{mmol}), \quad \alpha-$ methylcinnamaldehyde $(140 \mu \mathrm{L}, 1.00 \mathrm{mmol}, 1.0$ equiv) and $(S, S)-20 a(37 \mathrm{mg}, 0.10 \mathrm{mmol}, 0.1$ equiv) in $1 \mathrm{~mL}$ of $\mathrm{CH}_{2} \mathrm{Cl}_{2}$ for $10 \mathrm{~h}$, was obtained $344 \mathrm{mg}(0.82 \mathrm{mmol}, 82 \%)$ of $\mathbf{1 2 e}$ as a clear, viscous oil after column chromatography (pentane/ether, 3/1, $\mathrm{SiO}_{2}$ ). 
Data for $(s y n, s y n)-\mathbf{1 2 e}$ :

1슬 $: \quad\left(500 \mathrm{MHz}, \mathrm{CDCl}_{3}\right)$

$7.33(\mathrm{t}, J=7.3,2 \mathrm{H}, \mathrm{HC}(15)) ; 7.27(\mathrm{~d}, J=6.6,2 \mathrm{H}, \mathrm{HC}(14)) ; 7.21(\mathrm{t}, J=7.4,1$ H, HC(16)); 6.62 (br s, 1 H, HC(8)); 4.52 (br s, 1 H, HC(6)); 4.45 (sext, J = 5.9, 1 $\mathrm{H}, \mathrm{HC}(2)) ; 2.91$ (d, $J=2.9,1 \mathrm{H}, \mathrm{HO}) ; 2.86(\mathrm{qd}, J=7.3,3.6,1 \mathrm{H}, \mathrm{HC}(5))$; 2.842.62 (ABX, $2 \mathrm{H}, \mathrm{H}_{2} \mathrm{C}(3)$ ); 1.83 (d, $J=0.7,3 \mathrm{H}, \mathrm{H}_{3} \mathrm{C}(12)$ ); 1.23 (d, $J=6.1,3 \mathrm{H}$, $\left.\mathrm{H}_{3} \mathrm{C}(1)\right) ; 1.13$ (d, J= 7.3, $\left.3 \mathrm{H}, \mathrm{H}_{3} \mathrm{C}(11)\right) ; 1.06$ (m, $\left.21 \mathrm{H}, \mathrm{HC}(9), \mathrm{H}_{3} \mathrm{C}(10)\right)$

${ }^{13}$ C NMR: $\quad\left(126 \mathrm{MHz}, \mathrm{CDCl}_{3}\right)$

213.98 (C(4)); 137.61 (C(13)); 136.44 (C(7)); 128.99 (C(15)); 128.08 (C(14)); 126.38 (C(16)); 125.92 (C(8)); 75.01 (C(6)); 65.07 (C(2)); 51.75 (C(3)); 49.03 (C(5)); 24.12 (C(1)); 18.03 (C(10)); 15.25 (C(12)); 12.36 (C(9)); 9.42 (C(11))

IR: (neat)

3467 (br, m); 2943 (s); 2866 (s); 1705 (m); 1462 (m); 1375 (m); 1130 (m); 1097 (m); $1012(\mathrm{~m})$

MS: $\quad(\mathrm{FI})$

$418.2\left(\mathrm{M}^{+}, 55\right) ; 401.2$ (3); 375.2 (14); 337.1 (4); 272.2 (95); 229.1 (100); 201.3 (5); 146.1 (99)

Opt. Rot.: $\quad[\alpha]_{\mathrm{D}}^{24}+15.1(\mathrm{c}=2.2, \mathrm{EtOH})$

TLC: $\quad R_{f} 0.22$ (pentane/ether, 3/1) [silica gel, short uv]

SFC: $\quad t_{\mathrm{R}} 2.983 \mathrm{~min}(\mathrm{OD}, 150 \mathrm{bar}, 3 \mathrm{~mL} / \mathrm{min}, 15 \% \mathrm{MeOH})$

Analysis: $\quad \mathrm{C}_{25} \mathrm{H}_{42} \mathrm{O}_{3} \mathrm{Si}(418.68)$

$\begin{array}{lll}\text { Calcd: } & \text { C }, 71.72 ; & H, 10.11 \% \\ \text { Found: } & \text { C, } 71.46 ; & H, 10.04 \%\end{array}$

\section{References}

(1) Still, W. C.; Kahn, M.; Mitra, A. J. Org. Chem. 1978, 43, 2923.

(2) Gilman, H.; Cartledge, F. K. J. Organomet. Chem. 1964, 2, 447.

(3) Hall, P. L.; Gilchrist, J. H.; Collum, D. B. J. Am. Chem. Soc. 1991, 113, 9571.

(4) Taniguchi, Y.; Inanaga, J.; Yamaguchi, M. Bull. Chem. Soc. Jpn. 1981, 54, 3229.

(5) Busch-Petersen, J.; Corey, E. J. Tetrahedron Lett. 2000, 41, 2515.

(6) Cohen, F.; Overman, L. E. J. Am. Chem. Soc. 2001, 123, 10782. 